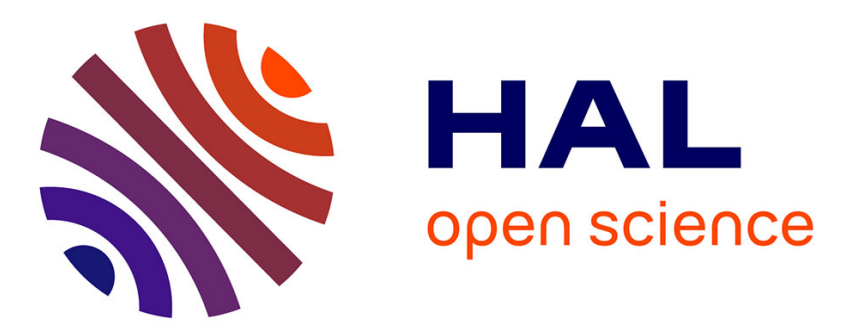

\title{
Spectral analysis of turbulence in anisothermal channel flows
}

Frederic Aulery, Dorian Dupuy, Adrien Toutant, Françoise Bataille, Ye Zhou

\section{To cite this version:}

Frederic Aulery, Dorian Dupuy, Adrien Toutant, Françoise Bataille, Ye Zhou. Spectral analysis of turbulence in anisothermal channel flows. Computers and Fluids, 2017, 151, pp.115 - 131. 10.1016/j.compfluid.2016.06.011 . hal-01906284

\section{HAL Id: hal-01906284 \\ https://hal-univ-perp.archives-ouvertes.fr/hal-01906284}

Submitted on 26 Oct 2018

HAL is a multi-disciplinary open access archive for the deposit and dissemination of scientific research documents, whether they are published or not. The documents may come from teaching and research institutions in France or abroad, or from public or private research centers.
L'archive ouverte pluridisciplinaire HAL, est destinée au dépôt et à la diffusion de documents scientifiques de niveau recherche, publiés ou non, émanant des établissements d'enseignement et de recherche français ou étrangers, des laboratoires publics ou privés. 


\title{
Spectral analysis of turbulence in anisothermal channel flows
}

\author{
Frederic Aulery ${ }^{\mathrm{a}, \mathrm{b}, *}$, Dorian Dupuy ${ }^{\mathrm{a}}$, Adrien Toutant $^{\mathrm{a}}$, Françoise Bataille ${ }^{\mathrm{a}}$, Ye \\ Zhou $^{\mathrm{c}}$ \\ ${ }^{a}$ PROMES CNRS, Université de Perpignan Via Domitia, Rambla de la thermodynamique, \\ Tecnosud, 66100 Perpignan, France \\ ${ }^{b}$ Univ. Orléans, INSA-CVL, PRISME, EA 4229, F45072, Orléans, France \\ ${ }^{c}$ Lawrence Livermore National Laboratory, Livermore 94550, USA
}

\begin{abstract}
In very anisothermal turbulent flows, the temperature gradient and turbulence are strongly coupled. The impact of the temperature gradient on turbulent kinetic energy (TKE) balance terms is of particular importance. It is investigated using direct numerical simulations and large eddy simulations of a fully developed anisothermal channel flow. A low Mach flow at two turbulent Reynolds number (180 and 395) is considered. The temperatures of the two channel walls are $293 \mathrm{~K}$ and $586 \mathrm{~K}$. Comparison with isothermal channel flows are carried out. The turbulent kinetic energy spectral evolution equation is established and is decomposed into the three distinctive mechanisms: production, nonlinear transfer and viscous effects. The decomposition isolates the terms that vanish in the isothermal case, namely purely anisothermal effects. The behavior of each term is first discussed in the isothermal case. The alteration of the TKE balance terms with the temperature gradient is then analysed relatively to the Reynolds number variation. The thermal gradient effect is characterized by the combined effect of local Reynolds number variation and the complex interaction between temperature and turbulence. The purely anisothermal contribution moves the energy from the hot side to the cold side and accounts for near $10 \%$ of the total process.
\end{abstract}

${ }^{*}$ Corresponding author : frederic.aulery@gmail.com 
Keywords: Turbulence, Temperature, Numerical simulations, Spectral analysis, Low Mach number flows

\section{Introduction}

Flows under strong temperature gradient are prevalent in many industrial processes. In particular, the conception of a high temperature solar receiver for concentrated solar power plants requires a better understanding of the physi5 cal effects of the temperature gradient on the turbulence [1]. These flows are characterized by a strong coupling between the energy equation and the momentum equation. Besides, high variations of the fluid properties associated with the temperature gradient are observed. As a result, these flows deviate from the behavior of incompressible flows [2]. An improved understanding of the energy transfers occurring in these flows would provide valuable informations to explain how the temperature gradient modify the flow. This would also benefit the development of turbulence models taking into account the specificities of these flows. Hence, we need reliable knowledge of the turbulent kinetic energy budget, that is of the mechanisms which governs the energy distribution: production, nonlinear transfer and viscous effects. The analysis of the aforementioned terms of the dynamic equation for the energy transfer process can be performed on the physical space or extended to the spectral space. The latter approach has been adopted in this study.

The analysis of turbulent kinetic energy assumes the decomposition of total kinetic energy, fundamental property of the flow, into two contributions: the mean kinetic energy, associated with the mean motion and the turbulent kinetic energy, associated with the turbulent motion. In the isothermal case, such decomposition of the total kinetic energy through Reynolds averaging is straightforward and brings no ambiguity about the physical interpretation of the terms [3]. Using this decomposition, the energy cascade process has been studied extensively for incompressible isothermal flows. The first studies on the subject were focused on homogeneous isotropic turbulence. For instance, Kol- 
mogorov's hypothesis on the locality of the energy transfer [4] and interacting scales $[5,6]$ have been validated by numerical simulations. Extension to the isothermal channel flows has been pursued by Domaradzki et al. [7], Marati et al. [8], Bolotnov et al. [9], and Cimarelli et al. [10, 11].

In the anisothermal case, the decomposition of total kinetic energy into mean and turbulent kinetic energy is a complex problem. The decomposition is not unique due to the variable density. This leads to several different definitions of turbulent kinetic energy. The most common decomposition uses densityweighted averaging, also called Favre averaging [12], to group total kinetic energy into two terms (see e.g. $[13,14]$ ). This method has also been referred by Chassaing et al. as binary regrouping [3]. This is opposed to the ternary regrouping proposed by Chassaing [15]. Another methodology uses a variable

40 change based on density square root weighted velocity. This variable change has been used by various authors in spatial and spectral space [16, 17, 18, 19, 20]. Regardless of the decomposition used, the spectral behaviour of anisothermal flow has so far received little attention from the litterature, though some interesting behaviours have been shown. For instance, the well-known Kolmogorov $45 \quad 5 / 3$ scaling law [21] falls short because of the strong dilatational effects of the temperature gradient [22]. The temperature gradient can in these flows be considered as a strong external agency that modifies the turbulence properties. This is analogous to flows subjected to strong magnetic field, rotation, or stratification [23, 24, 25]. Aulery et al. [16] have performed an analysis of the energy transfer processes with respect to the effect of the temperature gradient. This is to the best knowledge of the authors the only spectral study of the turbulent kinetic energy balance terms in this setting. However, the scope of the study is limited to a single Reynolds number.

We aim to enrich this study with further analysis of the influence of the turbulent Reynolds number on the role of the temperature gradient. Following Aulery et al. [16], turbulent kinetic energy spectral evolution equation is written using density square root weighted velocity. Our approach is based on the evaluation from numerical simulations of the terms of the dynamic equation 
for the energy transfer process in the case of a fully developed anisothermal channel flow. This geometry is one of the simpler that involves turbulence and high temperature gradient. Furthermore, the two homogeneous directions make the study of the energy transfer process easier. Direct numerical simulation and large eddy simulation are carried out to provide the data for this study. Knowledge of the instantaneous three-dimensional velocity flows is required to compute the various source terms of the energy transfer process, an information difficult to obtain using experimental investigations, limited by the measurement techniques, or closure theories, so far restricted to homogeneous isotropic turbulence.

The spectral equation for the turbulent kinetic energy and the decomposition used can be found in section 2. The detailed channel flow configuration is described in section 3 as well as the numerical method used and its validation in the isothermal case. Further details about the implementation of the turbulent kinetic energy evolution terms are also provided. Results are discussed in section 4 for the isothermal case and in section 5 for the anisothermal case.

\section{Momentum and spectral transport equations}

\subsection{Basic equations for direct numerical simulations}

In this study, a low Mach number approximation is considered. This approach is relevant when acoustics effects are negligible, an hypothesis valid for low Mach number (Ma $<0.3$ ). Following Paolucci [26], the method used relies on an asymptotic development of the variables of the Naviers-Stokes equations as a power series of the Mach number. Removing all but the smaller order terms leads to a new set of equations, free of acoustics constraints and CourantFriedrichs-Lewy condition [27]. The development also splits the pressure in two different terms:

- The thermodynamical pressure $P_{0}$ appears in the energy conservation equation and the equation of state. It is constant in space and represents the mean pressure in the domain. 
- The mechanical pressure $P$ appears in the momentum conservation equation and represents the pressure associated with momentum variations. It can vary spatially.

The gas considered is air; The effect of gravity is neglected and an ideal gas is considered.

These assumptions result in the following set of equations:

Mass equation:

$$
\frac{\partial \rho}{\partial t}+\frac{\partial\left(\rho U_{j}\right)}{\partial x_{j}}=0
$$

Momentum equation:

$$
\frac{\partial \rho U_{i}}{\partial t}+\frac{\partial \rho U_{j} U_{i}}{\partial x_{j}}=-\frac{\partial P}{\partial x_{i}}+\frac{\partial}{\partial x_{j}}\left[\mu\left(\frac{\partial U_{i}}{\partial x_{j}}+\frac{\partial U_{j}}{\partial x_{i}}\right)\right]-\frac{2}{3} \frac{\partial}{\partial x_{j}}\left(\mu \frac{\partial U_{j}}{\partial x_{j}}\right)
$$

Energy equation:

$$
\rho C_{p}\left(\frac{\partial T}{\partial t}+U_{j} \frac{\partial T}{\partial x_{j}}\right)=\frac{\partial P_{0}}{\partial t}+\frac{\partial}{\partial x_{j}}\left(\lambda \frac{\partial T}{\partial x_{j}}\right)
$$

Ideal gas law:

$$
P_{0}=\rho r T
$$

Thermodynamic pressure homogeneity:

$$
\frac{\partial P_{0}}{\partial x}=\frac{\partial P_{0}}{\partial y}=\frac{\partial P_{0}}{\partial z}=0
$$

In this set of equations, $\rho$ is the density, $T$ the temperature, $\mu$ the dynamic viscosity, $\lambda$ the thermal conductivity, $C_{p}$ the heat capacity at constant pressure, $r$ the ideal gas specific constant, $t$ the time, $P$ the mechanical pressure, $P_{0}$ the thermodynamic pressure, $U_{i}$ the $i$-th component of velocity and $x_{i}$ the Cartesian coordinate in $i$-direction. Einstein summation convention is used. The temporal evolution of the thermodynamical pressure $P_{0}$ is computed from the integration of equation (1c) using the ideal gas law (1d) and the fact that the thermodynamical pressure $P_{0}$ is constant in space (1e).

\subsection{Equations and models for large-eddy simulations}

In this study, direct numerical simulations (DNS) and large eddy simulations (LES) are performed. In the case of LES, the subgrid terms related to the 
convective terms of equations (1b) and (1c) are modeled. The non-linear terms occurring in the viscous terms and in the conductive heat fluxes terms are neglected. Let us denote $(\cdot)$ the spatial filtering operator and $(\widetilde{\cdot})$ the Favre filtering operator defined for an arbitrary quantity $\phi$ as [28]:

$$
\widetilde{\phi}=\frac{\overline{\rho \phi}}{\bar{\rho}}
$$

The filtered low Mach number set of equations used with regard to LES is:

$$
\begin{gathered}
\frac{\partial \bar{\rho}}{\partial t}+\frac{\partial \bar{\rho} \widetilde{U}_{j}}{\partial x_{j}}=0 \\
\frac{\partial \bar{\rho} \widetilde{U}_{i}}{\partial t}+\frac{\partial \bar{\rho} \widetilde{U_{j}} \widetilde{U_{i}}}{\partial x_{j}}=-\frac{\partial \bar{P}}{\partial x_{i}}+\frac{\partial}{\partial x_{j}}\left[\bar{\mu}\left(\frac{\partial \widetilde{U}_{i}}{\partial x_{j}}+\frac{\partial \widetilde{U}_{j}}{\partial x_{i}}\right)\right]-\frac{2}{3} \frac{\partial}{\partial x_{i}}\left(\bar{\mu} \frac{\partial \widetilde{U}_{j}}{\partial x_{j}}\right)-\frac{\partial \bar{\rho} \tau_{i j}}{\partial x_{j}} \\
C_{p}\left(\frac{\partial \bar{\rho} \widetilde{T}}{\partial t}+\frac{\partial \bar{\rho} \widetilde{U_{j}} \widetilde{T}}{\partial x_{j}}\right)=\frac{\partial \overline{P_{0}}}{\partial t}+\frac{\partial}{\partial x_{j}}\left(\bar{\lambda} \frac{\partial \widetilde{T}}{\partial x_{j}}\right)-\frac{\partial \bar{\rho} C_{p} \Im_{j}}{\partial x_{j}} \\
\frac{\partial \overline{P_{0}}}{\partial x}=\frac{\partial \overline{P_{0}}}{\partial y}=\frac{\partial \overline{P_{0}}}{\partial z}=0
\end{gathered}
$$

with:

$$
\begin{gathered}
\tau_{i j}=\widetilde{U_{j} U_{i}}-\widetilde{U_{j}} \widetilde{U_{i}} \\
\Im_{j}=\widetilde{U_{j} T}-\widetilde{U_{j} T} \widetilde{T}
\end{gathered}
$$

The WALE model [29] has been chosen for the modelling of the subgrid scale tensor $\tau_{i j}$. First, the subgrid scale viscosity $\nu_{s g}$ is computed using:

$$
\nu_{s g}=\left(C_{w} \widetilde{\Delta}\right)^{2} \frac{\left(s_{i j}^{d} s_{i j}^{d}\right)^{3 / 2}}{\left(\widetilde{S}_{i j} \widetilde{S}_{i j}\right)^{5 / 2}+\left(s_{i j}^{d} s_{i j}^{d}\right)^{5 / 4}}
$$


with $\widetilde{\Delta}$ the subgrid characteristic length scale, $C_{w}$ a constant, $\widetilde{S}_{i j}$ the filtered strain tensor:

$$
\widetilde{S}_{i j}=\frac{1}{2}\left(\frac{\partial \widetilde{U}_{i}}{\partial x_{j}}+\frac{\partial \widetilde{U}_{j}}{\partial x_{i}}\right)
$$

and $s_{i j}^{d}$ the traceless symmetric part of the square of the velocity gradient tensor which can be written as:

$$
s_{i j}^{d}=\widetilde{S}_{i k} \widetilde{S}_{k j}+\widetilde{\Omega}_{i k} \widetilde{\Omega}_{k j}-\frac{1}{3} \delta_{i j}\left(\widetilde{S}_{m n} \widetilde{S}_{m n}-\widetilde{\Omega}_{m n} \widetilde{\Omega}_{m n}\right)
$$

where $\widetilde{\Omega}_{i j}$ is the filtered rotation vector:

$$
\widetilde{\Omega}_{i j}=\frac{1}{2}\left(\frac{\partial \widetilde{U}_{i}}{\partial x_{j}}-\frac{\partial \widetilde{U}_{j}}{\partial x_{i}}\right)
$$

The deviatoric part $\tau_{i j}^{d}$ of the subgrid scale tensor is then calculated with the following expression:

$$
\tau_{i j}^{d}=\tau_{i j}-\frac{1}{3} \delta_{i j} \tau_{k k}=-2 \nu_{s g} \widetilde{S}_{i j}
$$

The complementary term $\frac{1}{3} \delta_{i j} \tau_{k k}$ is added to the filtered pressure term and requires no modelling.

The subgrid scale heat flux $\Im_{j}$ is calculated using a subgrid scale diffusivity model [30]:

$$
\Im_{j}=\kappa_{s g} \frac{\partial \widetilde{T}}{\partial x_{j}}
$$

with $\kappa_{s g}$ the subgrid scale diffusivity, linked to the subgrid scale viscosity through the subgrid scale Prandtl number $P r_{s g}=\frac{\nu_{s g}}{\kappa_{s g}}$.

To compute dynamic viscosity, Sutherland law [31] has been adopted:

$$
\mu(T)=\mu_{0}\left(\frac{T}{T_{0}}\right)^{\frac{3}{2}} \frac{T_{0}+S}{T+S}
$$

with $\mu_{0}=1.716 \cdot 10^{-5}, S=110.4$ and $T_{0}=273.15$. Thermal conductivity is deduced from Prandtl number, assumed constant and equal to 0.7:

$$
\lambda(T)=\frac{C_{p}}{\operatorname{Pr}} \mu(T)
$$

The heat capacity at constant pressure is $C_{p}=1005 \mathrm{~J} \mathrm{~kg}^{-1} \mathrm{~K}^{-1}$. The ideal gas specific constant is $r=287 \mathrm{~J} \mathrm{~kg}^{-1} \mathrm{~K}^{-1}$. 


\subsection{Turbulent kinetic energy equation}

We use a change of variable, introduced by Cook and Zhou [20] and Kida and Orszag [18], suitable for the study of flows with an highly variable density. Firstly, we focus on the left side of the low Mach moment equation (1b):

$$
\frac{\partial \rho U_{i}}{\partial t}+\frac{\partial \rho U_{i} U_{j}}{\partial x_{j}}
$$

Introducing the new variables $V_{i}=\rho^{\frac{1}{2}} U_{i}$ and $b=\rho^{-\frac{1}{2}}$ into (13), we get:

$$
\begin{aligned}
\frac{\partial \rho U_{i}}{\partial t}+\frac{\partial \rho U_{i} U_{j}}{\partial x_{j}} & =\frac{\partial \sqrt{\rho} V_{i}}{\partial t}+\frac{\partial \sqrt{\rho} V_{i} U_{j}}{\partial x_{j}} \\
& =\sqrt{\rho} \frac{\partial V_{i}}{\partial t}+\frac{1}{2} b V_{i} \frac{\partial \rho}{\partial t}+\sqrt{\rho} \frac{\partial V_{i} U_{j}}{\partial x_{j}}+\frac{1}{2} V_{i} U_{j} b \frac{\partial \rho}{\partial x_{j}} \\
& =\sqrt{\rho}\left(\frac{\partial V_{i}}{\partial t}+\frac{\partial V_{i} U_{j}}{\partial x_{j}}\right)+\frac{1}{2} b V_{i}\left(\frac{\partial \rho}{\partial t}+U_{j} \frac{\partial \rho}{\partial x_{j}}\right)
\end{aligned}
$$

From the mass equation (1a), we can write:

$$
\frac{\partial \rho}{\partial t}+U_{j} \frac{\partial \rho}{\partial x_{j}}=-\rho \frac{\partial U_{j}}{\partial x_{j}}
$$

Now, by substituting (15) into (14):

$$
\begin{aligned}
\frac{\partial \rho U_{i}}{\partial t}+\frac{\partial \rho U_{i} U_{j}}{\partial x_{j}} & =\sqrt{\rho}\left(\frac{\partial V_{i}}{\partial t}+\frac{\partial V_{i} U_{j}}{\partial x_{j}}\right)+\frac{1}{2} b V_{i}\left(-\rho \frac{\partial U_{j}}{\partial x_{j}}\right) \\
& =\frac{1}{b}\left(\frac{\partial V_{i}}{\partial t}+\frac{\partial V_{i} U_{j}}{\partial x_{j}}-\frac{1}{2} V_{i} \frac{\partial U_{j}}{\partial x_{j}}\right)
\end{aligned}
$$

Finally, the variable change leads to the following momentum equation:

$\frac{\partial V_{i}}{\partial t}+\frac{\partial V_{i} U_{j}}{\partial x_{j}}-\frac{1}{2} V_{i} \frac{\partial U_{j}}{\partial x_{j}}=b\left[-\frac{\partial P}{\partial x_{i}}+\frac{\partial}{\partial x_{j}}\left[\mu\left(\frac{\partial U_{i}}{\partial x_{j}}+\frac{\partial U_{j}}{\partial x_{i}}\right)\right]-\frac{2}{3} \frac{\partial}{\partial x_{i}}\left(\mu \frac{\partial U_{j}}{\partial x_{j}}\right)\right]$

Following Aulery et al. [16], the turbulent kinetic energy evolution can be decomposed into three main mechanisms which are the turbulent production $\Pi$ defined by equation (19a), the total transfer $\sum_{n} T_{n}$ which is the sum of all triadic and pressure terms and finally the viscous part $D(19 \mathrm{~g})$ that brings together all viscous effects.

$$
\frac{\partial E_{c}(k, y, t)}{\partial t}=\Pi(k, y, t)+\sum_{n} T_{n}(k, y, t)+D(k, y, t)
$$

110

A derivation of the spectral turbulent kinetic energy evolution equation is presented in Appendix A. 


\subsection{Decomposition of TKE evolution terms}

For our study, we choose to decompose transfer mechanisms using a Bolotnov et al. [9] based decomposition. The triadic and pressure part are split following the gradient direction. The terms with derivatives in the homogeneous $x O z$ plane $\left(\frac{\partial}{\partial x}\right.$ and $\left.\frac{\partial}{\partial z}\right)$ are named in-plane terms. The terms with derivatives in the wall-normal direction $\left(\frac{\partial}{\partial y}\right)$ are named inter-plane terms. In addition, we take into account the variation of scalar quantities due to temperature that results from the non-free divergence, the mean wall-normal velocity $\left\langle U_{y}\right\rangle$ and density gradient. These terms are null for isothermal incompressible flow and are stacked in one thermal effects term (19f). These denominations are derived from the work of Bolotnov et al. [9]. It is worth noting that these notations are linked to the mathematical writing of the term and may differ from physical behaviours.

Turbulent production

$$
\Pi=\int_{k}-\Re\left[{\widehat{v_{x}^{\prime}}}^{*} \widehat{u_{y}^{\prime}} \frac{\partial\left\langle V_{x}\right\rangle}{\partial y}\right] d \underline{k}
$$

Triadic in-plane term

$$
T_{i n}=\int_{k}-\Re\left[\sum_{i} \sum_{j=x, z} \widehat{v_{i}^{\prime}} \frac{\partial \hat{v}_{i}^{\prime} u_{j}^{\prime}}{\partial x_{j}}\right] d \underline{k}
$$

Triadic inter-plane term

$$
T_{i t}=\int_{k}-\Re\left[\sum_{i}{\widehat{v_{i}^{\prime}}}^{*} \frac{\partial \widehat{v_{i}^{\prime} u_{y}^{\prime}}}{\partial y}\right] d \underline{k}
$$

Pressure in-plane term

$$
\Phi_{i n}=\int_{k}-\Re\left[\sum_{i=x, z}{\widehat{v_{i}^{\prime}}}^{*}\left(b \widehat{\frac{\partial P}{\partial x_{i}}}\right)\right] d \underline{k}
$$

Pressure inter-plane term

$$
\Phi_{i t}=\int_{k}-\Re\left[\widehat{v_{y}^{\prime}} *\left(\widehat{b \frac{\partial P}{\partial y}}\right)\right] d \underline{k}
$$


Thermal effect term

$$
\begin{aligned}
\Gamma=\int_{k}-\Re\left[\sum_{i}\right. & \left(\widehat{v_{y}^{\prime}} \widehat{u}_{y}^{\prime} \frac{\partial\left\langle V_{y}\right\rangle}{\partial y}+\left\langle U_{y}\right\rangle \frac{\partial \frac{1}{2}{\widehat{v_{i}^{\prime}}}^{*} \widehat{v_{i}^{\prime}}}{\partial y}+\frac{1}{2} \widehat{v_{i}^{\prime}} \widehat{v_{i}^{\prime}} \frac{\partial\left\langle U_{y}\right\rangle}{\partial y}\right. \\
& \left.\left.-\sum_{j} \frac{1}{2}{\widehat{v_{i}^{\prime}}}^{*}\left(\widehat{v}_{i}^{\prime} \frac{\widehat{\partial u_{j}^{\prime}}}{\partial x_{j}}\right)+\sum_{j} \frac{1}{2}{\widehat{v_{i}^{\prime}}}^{*}\left\langle V_{i}\right\rangle \frac{\widehat{\partial u_{j}^{\prime}}}{\partial x_{j}}\right)\right] d \underline{k}
\end{aligned}
$$

Viscous effect

$$
D=\int_{k} \Re\left[{\widehat{v_{i}^{\prime}}}^{*} F_{k}^{y}\left\{b \frac{\partial}{\partial x_{j}}\left[\mu\left(\frac{\partial U_{i}}{\partial x_{j}}+\frac{\partial U_{j}}{\partial x_{i}}\right)\right]-b \frac{2}{3} \frac{\partial}{\partial x_{i}}\left(\mu \frac{\partial U_{j}}{\partial x_{j}}\right)\right\}\right] d \underline{k}
$$

125

In the following sections, the averaged kinetic energy $\left\langle E_{c}\right\rangle=\frac{1}{2}\left\langle{\widehat{v_{i}^{\prime}}}^{*} \widehat{v_{i}^{\prime}}\right\rangle$, statistical average of the instantaneous kinetic energy $E_{c}$, will be studied. This quantity is independent of time. Its evolution equation can be written as:

$\frac{\partial\left\langle E_{c}\right\rangle(k, y)}{\partial t}=\langle\Pi\rangle(k, y)+\left\langle T_{i n}\right\rangle(k, y)+\left\langle T_{i t}\right\rangle(k, y)+\left\langle\Phi_{i n}\right\rangle(k, y)+\left\langle\Phi_{i t}\right\rangle(k, y)+\langle\Gamma\rangle(k, y)+\langle D\rangle(k, y)=0$

Consequently, the statistical average of each term of equation (19) will be analysed and computed.

\section{Simulated datasets and validation}

\subsection{Channel flow configuration} $L_{x}=4 \pi h, L_{y}=2 h$ and $L_{z}=2 \pi h$ with $h=15 \mathrm{~mm}$.

Because of the temperature gradient, the turbulent Reynolds numbers $R e_{\tau}$ at each sides of the channel are different, with the turbulent Reynolds number at 


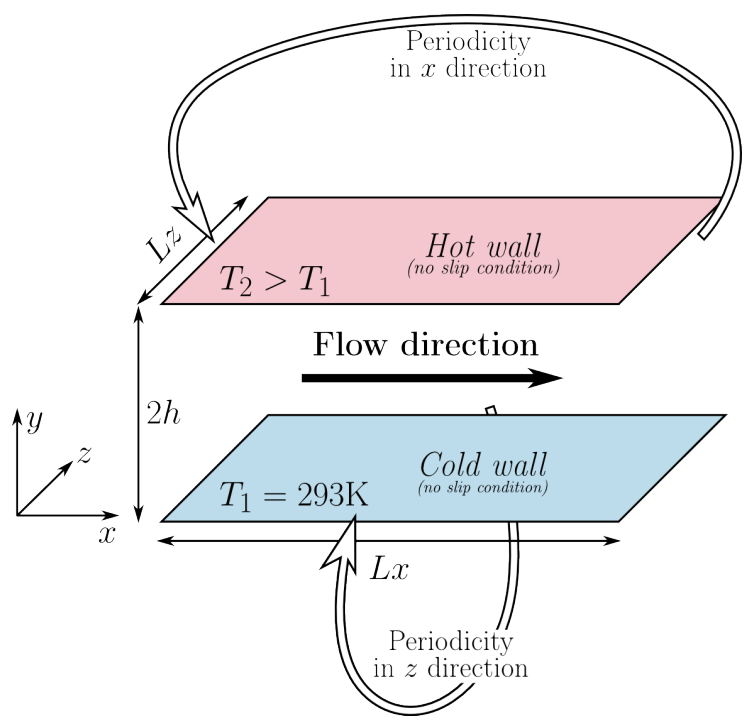

Figure 1: Biperiodic anisothermal channel flow

the cold side being greater. Thus, the mean turbulent Reynolds number $R e_{\tau}^{m}$ is defined as the average of the turbulent Reynolds number calculated at the cold side and at the hot side. Simulations were carried out in various configurations as described in table 1 . In this table, the number of grid points in the direction $i$ is denoted $N_{i}$ and the cell size in wall-units $\Delta i^{+}$. The wall-units scaling is based on the turbulent Reynolds number of the cold side, since it is more restrictive.

Two mean turbulent Reynolds number were selected (180 and 395) as well as temperature ratios of 2 and 1 for the incompressible limit case. Hence, four configurations are simulated in total. The computational cost increase with Reynolds number restricts the mesh refinement at $R e_{\tau}^{m}=395$ compared to the simulations at $R e_{\tau}^{m}=180$. As the temperature gradient induces a local Reynolds number increase, the simulations performed are more precise in the isothermal case for similar reasons. The cell sizes in wall-units in the wall-normal direction is increased by 0.25 from an isothermal simulation to the anisothermal equivalent and 0.5 from low Reynolds number to high Reynolds number.

Since the simulations at $R e_{\tau}^{m}=180$ are less expensive in terms of com- 


\begin{tabular}{|c|c|c|c|c|c|c|c|c|c|c|c|}
\hline Name & Type & $R e_{\tau}$ & $T_{r}$ & $L_{x}$ & $L_{z}$ & $\Delta x^{+}$ & $\Delta z^{+}$ & $\Delta y^{+}$ & $N_{x}$ & $N_{y}$ & $N_{z}$ \\
\hline $180-1$ & DNS & 184 & 1 & $4 \pi h$ & $2 \pi h$ & 6 & 3 & $0.25-2.3$ & 382 & 300 & 382 \\
\hline $180-2$ & DNS & $270-108$ & 2 & $4 \pi h$ & $2 \pi h$ & 17.6 & 5.9 & $0.5-7.8$ & 192 & 140 & 288 \\
\hline $395-1$ & LES & 395 & 1 & $4 \pi h$ & $2 \pi h$ & 11 & 11 & $0.75-7.5$ & 448 & 200 & 224 \\
\hline $395-2$ & LES & $542-221$ & 2 & $4 \pi h$ & $2 \pi h$ & 14 & 14 & $1-7.5$ & 448 & 200 & 224 \\
\hline
\end{tabular}

Table 1: List of simulations and mesh parameters.

putational cost, we were able to capture the fine flow behavior using direct numerical simulation. This contrasts with the simulations at $R e_{\tau}^{m}=395$. At high Reynolds number, the calculation power and mesh sizes required for a full DNS would be excessive. Large eddy simulation appears as the preferable alternative. With the subgrid scales modelling, the simulations can yield accurate statistics despite coarser meshes relatively to the turbulent Reynolds number. Therefore, DNS is used at $R e_{\tau}^{m}=180$ and LES at $R e_{\tau}^{m}=395$.

\subsection{Numerical set up}

The resolution of the low Mach equations involves a finite volume solver. A third order Runge-Kutta is used for time derivatives. A fourth order centered scheme is used for momentum convection. A third order QUICK scheme (quadratic upstream interpolation for convective kinetics [32]) is used for temperature convection. TrioCFD is used to perform the simulations [33].

As there is no mean pressure gradient in the channel flow because of periodic boundary conditions, a streamwise volume force $F$ is added to keep the mass flow rate constant. For both the DNS and the LES, the driving force is regulated through the masse flow rate second derivative:

$$
F_{t+1}=F_{t}+C_{0} \frac{D_{\text {target }}-2 D_{t}+D_{t-1}}{\Delta t}
$$

with $C_{0}$ a damping constant, $D$ the mass flow rate, $D_{\text {target }}$ the targeted mass flow rate and $t-1, t$ and $t+1$ indices related to the previous, current and next time step respectively. Walls have no slip condition. The initial velocity 
field is parabolic in the streamwise direction and sinusoidal in the wall-normal direction and permits to initiate the turbulence.

Four meshes were adopted as shown in table 1. Each mesh is regular in both homogeneous directions. The grid points in the wall-normal direction follow a hyperbolic tangent transformation:

$$
y_{k}=L_{y}\left(1+\frac{1}{a} \tanh \left[\left(\frac{k-1}{N_{y}-1}-1\right) \tanh ^{-1}(a)\right]\right)
$$

where $a$ is a constant associated with the mesh dilatation. The precision increase in the boundary layer compared to an uniform mesh allows the use of the no-slip boundary condition.

\subsection{Data collection and filtering for spectral analysis}

The data required to compute the different terms of equation (19) is acquired during the simulation. The terms of equation (19) are calculated at each wallnormal coordinate at particular time steps then time averaged. A time-sampling corresponding to 20 timesteps was used. The time average is performed over a total duration of $12.3 t_{c}$, where $t_{c}=h / U_{\tau}$ is the characteristic timescale of the simulation. This is sufficient for the spectral statistics to converge. The Fourier transform is numerically computed as:

$$
\widehat{g}\left(k_{x, m}, y, k_{z, n}\right)=\frac{1}{N_{x} N_{z}} \sum_{p=0}^{N_{x}-1} \sum_{k=0}^{N_{z}-1} g\left(x_{p}, y, z_{k}\right) \exp \left(-2 \pi i\left(\frac{m p}{N_{x}}+\frac{k n}{N_{z}}\right)\right)
$$

This is done using the FFTW C subroutine library [34].

A low pass filter is applied to the spectral data. This could be done because a clear separation between high frequency noise and the wanted signal appears in the Fourier transform of the spectral data. This can be seen as a movingaverage that takes into account $2 \mathrm{D}$ wavenumber vectors of nearby norms. The energy transfer processes are direction-dependant, that is not identical in $x$ and $z$ directions. It is thus of great importance that this averaging does not favor a

particular direction. 


\subsection{Validation of the mean field profiles}

The numerical method described above has been validated with regard to DNS using the results of Kim et al. [35] in the isothermal case. The simulation performed by Kim et al. [35] has a turbulent Reynolds number of 180. It has been compared to our DNS in the same conditions in figures 2 and 3. The following standard scaling is used:

$$
\begin{gathered}
y^{+}=R e_{\tau} \frac{y}{h} \\
U^{+}=\frac{\langle U\rangle}{U_{\tau}}
\end{gathered}
$$

where $U_{\tau}$ is the wall friction velocity. Similarly to (24b), each RMS quantity in figure 3 is scaled by the friction velocity $U_{\tau}$ and the velocity correlation by $U_{\tau}^{2}$. The two simulations give nearly identical results for both mean and with the results of Moser et al. [36] in the isothermal case at $R e_{\tau}=395$ (not shown here). This validates our numerical method at the incompressible limit for both DNS and LES.

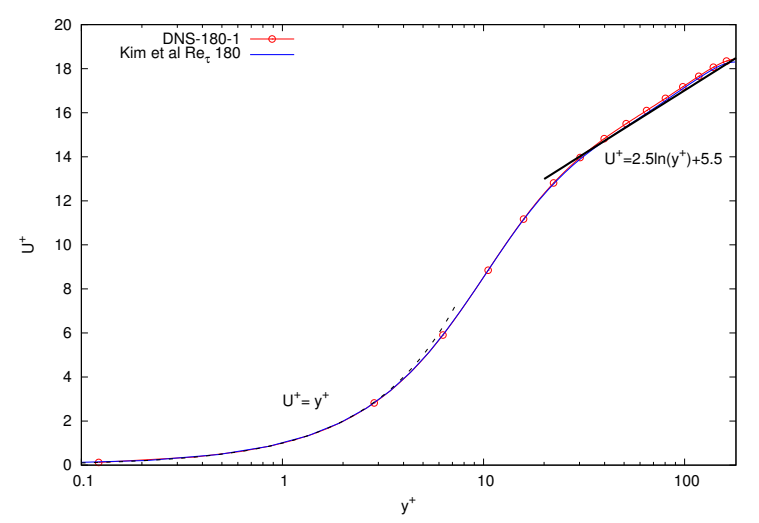

Figure 2: Mean longitudinal velocity comparison with direct numerical simulation of Kim et al. [35] at $R e_{\tau}=180$. 


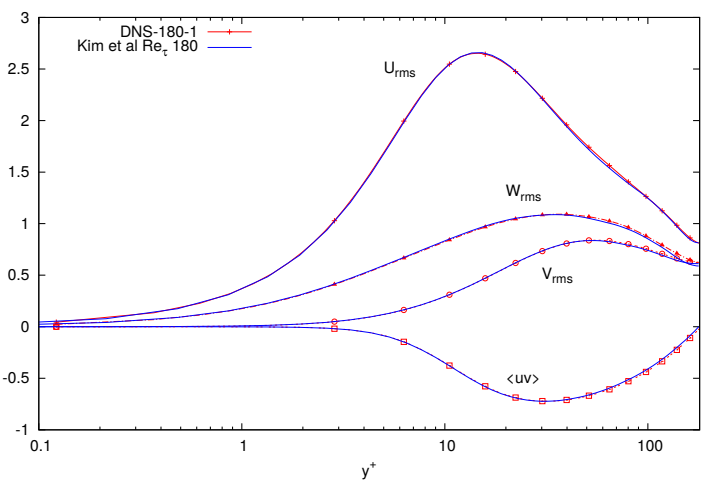

Figure 3: Velocity fluctuations comparison with direct numerical simulation of Kim et al. [35] at $\operatorname{Re}_{\tau}=180$.

\subsection{Validation of the spectra measurements}

As written in 2.4, the TKE evolution equation decomposition follows Bolotnov et al. [9] decomposition and allows comparison on isothermal flow spectra. Figure 4 compares the turbulent production (equation (19a)), figure 5 the inplane triadic transfer term (equation (19b)) and figure 6 the inter-plane transfer term (equation (19c)). All these figures use the same normalisation as Bolotnov and the colors scales are identical. The following scaling is used for the wave number:

$$
k^{+}=\frac{k h}{R e_{\tau}}
$$

The comparison reveals that our results match with those of Bolotnov with respect to magnitude and global shape for all terms. The turbulent production (figure 4$)$ is centered on the same area $\left(0.056<k^{+}<1\right.$ and $\left.10<y^{+}<17\right)$ and decreases at the same rate.

For the in-plane triadic transfer (figure 5), our results are in agreement with the two major areas found by Bolotnov: one negative centered on $k^{+}=0.08$ and $y^{+}=12$, and one positive centered on $y^{+}=18$ and $k^{+}=0.178$. A low magnitude positive area appears in our simulation around $k^{+}=0.02$ and $y^{+}=13$ whereas the figure of Bolotnov suggests that the term tends to zero 


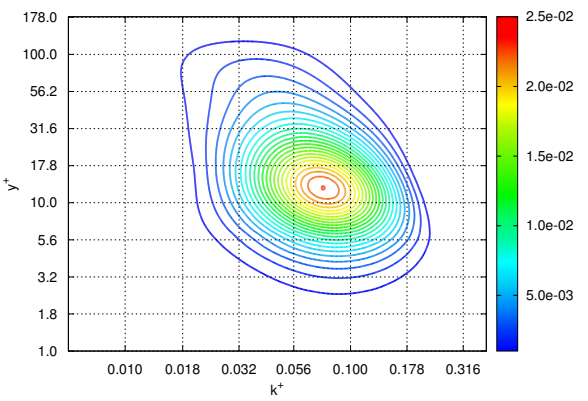

(a) DNS $R e_{\tau}=180$ isothermal

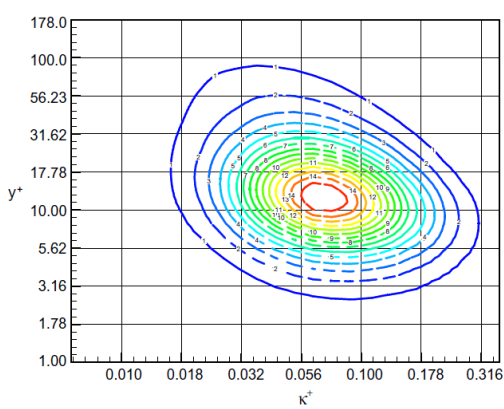

(b) Bolotnov et al.

Figure 4: Comparison of turbulent production spectra.

\section{Isothermal energy transport of distinctive Reynolds numbers}

reported in figures 7 to 17 . For each set, a vertical reading gives a thermal 


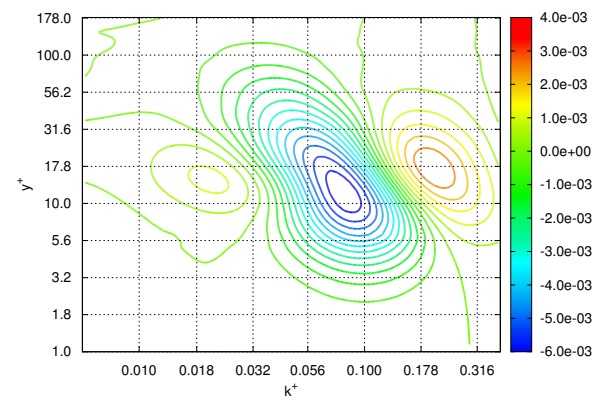

(a) DNS $R e_{\tau}=180$

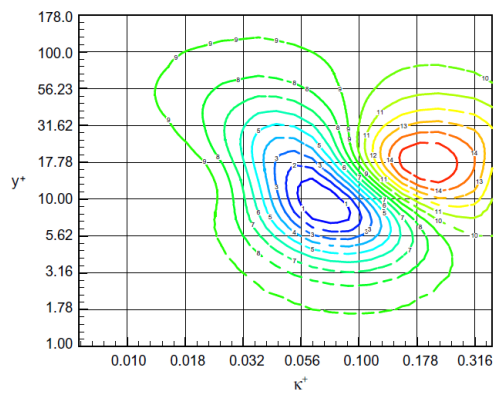

(b) Bolotnov et al.

Figure 5: Comparison of in-plane triadic transfer spectra.

comparison (isothermal case, cold and hot sides for the anisothermal case) at a constant Reynolds number, while a horizontal reading gives a comparison between Reynolds Number for a same thermal configuration. Figure 7 and figures $8-17$ are scaled by $U_{\tau} \nu$ and by the maximum of production in the isothermal case for each Reynolds number, respectively. The wave number is scaled with the half-height of the channel $h$. The analysis done in this section requires only the middle panel of figures 7 to 17 .

\subsection{Isothermal turbulent spectra at low Reynolds number}

At first, the turbulent kinetic energy is plotted on figure 7(c). As expected, the maximum of TKE is around $y^{+}=11$ for the larger scales $(k h<2)$. Compared to the high scales, the energy of the small scales is divided by more than $10^{10}$. This ensures the capture of the essential phenomena from the largest scale to the smallest. The significant parts of TKE and transfer mechanisms are not filtered in the case of LES, as they are located beyond the cutoff frequency which is around $k h=20$ (the filter is only visible at high Reynolds number).

The TKE production (figure $8(\mathrm{c})$ ) is limited at $k h<4$ in term of the spectral scales and at $y^{+}>5$ in term of the wall distance. The maximum of the production is close to the maximum of TKE. The scale range of the production 


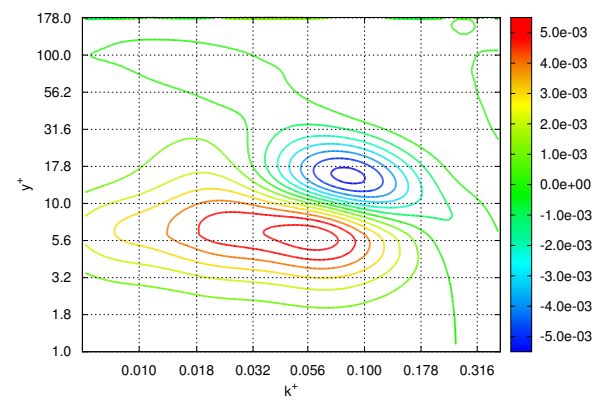

(a) DNS $R e_{\tau}=180$

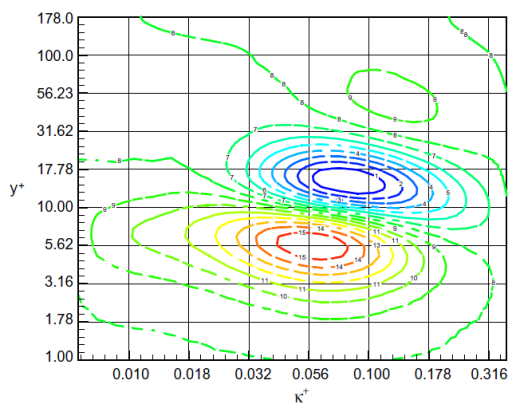

(b) Bolotnov et al.

Figure 6: Comparison of inter-plane triadic transfer spectra.

240

tends to zero near $y^{+}=100$.

The total energy transfer (figure $9(\mathrm{c})$ ) is characterized by an inverse cascade from intermediary spectral scales to the large scales. The intermediary scales where the energy is removed are located near the production term peak area. Note that the size of structures receiving energy is longer than the channel size $(k h>1)$. The energy transfer occurs between scales with a scale ratio greater than two, which is characteristic of non-local transfers [40]. In global point of view, the energy is transferred closer to the wall, a mechanism removing the energy to the streaks.

As expected, the viscous effect (figure 10(c)) balances the total energy transfer mechanism. The energy is taken from the viscous layer at large scales and tends to zero at the small scales. This may correspond to the friction created by the streaks close to the wall.

${ }_{255}$ The total energy transfer is the sum of all triadic and pressure terms. Let us now study them individually

The triadic in-plane term (figure 11(c)) is composed of three areas around $y^{+}=20$. The first and last areas are positive with smaller $y^{+}$ranges but the 
middle one is negative with larger spectral and $y^{+}$ranges. This term, therefore,

\subsection{Reynolds number effects on isothermal turbulent spectra}

The impact of Reynolds number variations on the turbulent kinetic energy balance terms will be investigated using the isothermal results at $R e_{\tau}=180$ and $R e_{\tau}=395$.

To begin with, the turbulent kinetic energy and TKE evolution terms tend to have wider wave space and $y^{+}$ranges at high Reynolds number. At the same time, the mechanisms are more distributed in the spectral space. The spatial extent of production stretches both into the central channel area and closer 
to the wall at $R e_{\tau}=395$ when compared to the $R e_{\tau}=180$ case, increasing

from $5<y^{+}<50$ (figure $8(\mathrm{c})$ ) to $3<y^{+}<300$ (figure $8(\mathrm{~d})$ ). Its spectral space extent has also increased from $0.7<k h<3$ to $0.4<k h<6$. Similar behaviour is observed for total transfer, in-plane and inter-plane triadic and pressure transfer terms.

Meanwhile, the turbulent scales shift with wall distance at high Reynolds number. The operative scales are larger far from the wall and smaller closer to the wall. The negative area range of the in-plane pressure transfer term (figure 14) is $0.4<k h<1$ at $y^{+}=200$ and $1<k h<2$ near $y^{+}=60$. Such scale shift is however not present at $R e_{\tau}=180$. The scale shift increases with Reynolds number is followed by all transfer terms and production.

It may be also be observed that the magnitude of the pressure transfer term as a proportion of the total transfer is lower at $R e_{\tau}=395$. This made the pressure relatively (but not physically) less important than triadic effect.

Finally, the turbulent scales involved in the transfer are less selected at high Reynolds number. At low Reynolds number, the in-plane triadic transfer (figure 305 11(c)) occurs in 3 very localized areas. Conversely, at high Reynolds number, the areas are no more separated in the wavenumber space (figure 11(d)). This is also true for triadic inter-plane transfer term and the total transfer term.

\section{Anisothermal turbulent spectra of distinctive Reynolds numbers}

\subsection{Anisothermal turbulent spectra at low Reynolds number}

In this subsection, the effect of thermal gradient is described by comparing an anisothermal flow at $R e_{\tau}=180$ against that from the isothermal simulation in last section. We will defer the investigation of the Reynolds number variations in an anisothermal setting to the next subsection.

The thermal effect term (figure 17, left panel), which is negligible in isothermal simulations, now has the same magnitude as the pressure transfer terms ( $\sim 10 \%$ of total transfer term). This term is negative at the hot side and positive at the cold side. This can be seen as an energy transfer from hot to cold 
side. One explanation is based on the mean vertical velocity, which is directed to the cold side. In the termal effect term (equation (19f)), one gets the advec320 tion of turbulent kinetic energy by the wall-normal velocity $\left\langle U_{y}\right\rangle \frac{\partial \frac{1}{2} \widehat{v}_{i}^{*} \widehat{v}_{i}^{\prime}}{\partial y}$. This term transfers TKE away from the wall at the hot side and towards the wall at the cold side.

Recall that the local Reynolds number is directly impacted by the thermal gradient. It is interesting that the terms at the hot side have larger magnitude than those at the cold side. The opposite behaviour would have been expected as the temperature gradient reduces the local Reynolds number at the hot side. Particular care should be taken in the interpretation of these amplitudes, as these differences do not necessarily apply to the total integrated values. On many terms, the hot side has a stronger magnitude than the cold side, but 330 due to the lower wave number range of active areas the total energy handled is actually lower than at the cold side. We can observe this phenomenon for the triadic inter-plane transfer (figures 12(a) and 12(e)). The hot side negative area has a two times larger amplitude than the cold side. At the same time, the negative area with maximum values has a very small wave number range at the hot side and a large one at the cold side.

The in-plane pressure term (figures 14(a) and 14(e)) is heavily modified by the temperature gradient. The behaviour of the hot side is similar to the isothermal case with a positive area close to the wall and a negative area near the center of the channel. At the cold side, an additional positive area appears at large scales near $y^{+}=100$. This asymmetry between the two sides of the channel is not present in the inter-plane pressure term (figures 15(a) and 15(e)). The inter-plane presure term is larger than its in-plane counterpart.

The total pressure term is modified as well (figures 16(a) and 16(e)). The positive area located near $y^{+}=60$ in the isothermal case (figure 16(c)) has 345 vanished at the hot side (figure 16(e)), leaving just a negative one from $y^{+}=10$ to the center of the channel. Conversely, at the cold side of the channel (figure 16(a)), the positive area is intensified in magnitude and increased in size.

Finally, the thermal gradient induces a Reynolds number variation between 
the flows at different sides of the channel. Compared to the cold side, the terms at the hot side are contracted, losing small scales. This is easily observable on the in-plane triadic term (figures 11(a) and 11(e)). For the TKE spectra, the small scales at the hot side have lower values while the large scales have the same energy level as that at the cold side. The shorter spectral range in the hot side is a direct consequence of a reduced local Reynolds number.

\subsection{Reynolds number effects on anisothermal turbulent spectra}

We now consider the effects of Reynolds number variations on the turbulent kinetic energy balance terms using the anisothermal results at both $R e_{\tau}=180$ and $R e_{\tau}=395$. The impact of the Reynolds number at both sides of the channel as well as the interplay between the temperature gradient and the mean Reynolds number must be considered.

The significant influence of increased Reynolds number is evident when the left and right panels of Figs. 7 - 17 are compared. At high Reynolds number, the anisothermal spectra largely experience a combined effect from the temperature gradient and the Reynolds number. The amplitudes of TKE evolution terms are intensified at both sides of the channel by the temperature gradient. The amplitudes are often far larger in the anisothermal spectra than their isothermal counterpart.

In many cases, the wave space and spatial extents of the TKE evolution terms also seem to increase with the temperature gradient. Such analysis should be taken with care, as terms are not more distributed in wave number space but just appear larger due to the higher amplitude.

The triadic in-plane term (figures 11(b) and 11(f)) evolves slightly differently with Reynolds number in the isothermal and anisothermal case. The positive area at large scales disappears almost completely in the isothermal case, whereas it remains significant in the anisothermal case.

Even at the $R e_{\tau}=395$ case, the near wall region of the hot and cold sides of the in-plane triadic transfer and of the total pressure term have a different behaviour. In the in-plane triadic term, the near wall negative area is greater 
at the cold side than at the hot side. In the total pressure term, the near wall positive area is greater at the hot side than at the cold side.

\section{Conclusion}

In this paper, we studied the effect of a strong temperature gradient on turbulent kinetic energy transfer processes in a fully developed channel flow. The analysis uses a variable change based on density square root weighted veloctransfer moves energy from the hot side to the cold side following the mean 
vertical velocity.

\section{Acknowledgment}

The authors gratefully acknowledge the CEA for the development of the TRUST platform. This work was granted access to the computer resources of CINES under the allocation 2014-c20142a5099 and 2015-c20152a5099 made by GENCI. The last author would like to dedicate this work to Dr. Chuck Leith in respectful acknowledgement of his exemplary scientific career. 


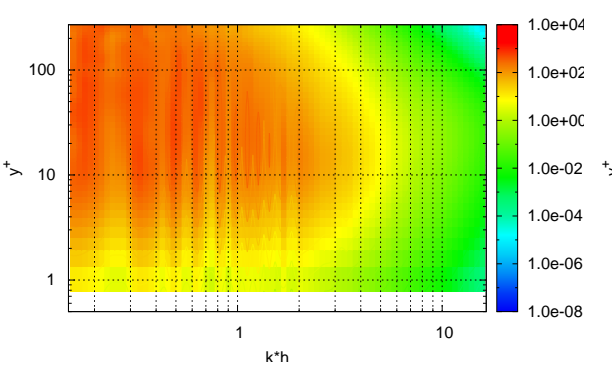

(a) Cold side $R e_{\tau}^{m}=180$

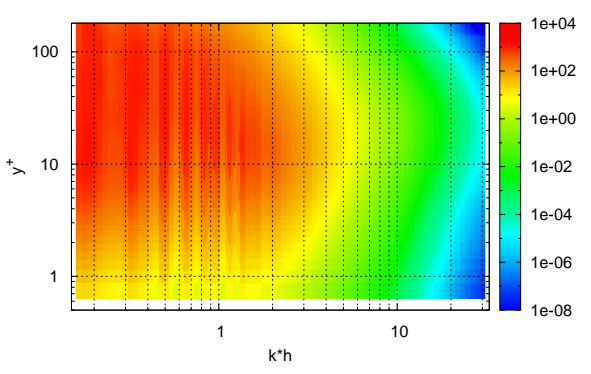

(c) Isothermal $R e_{\tau}=180$

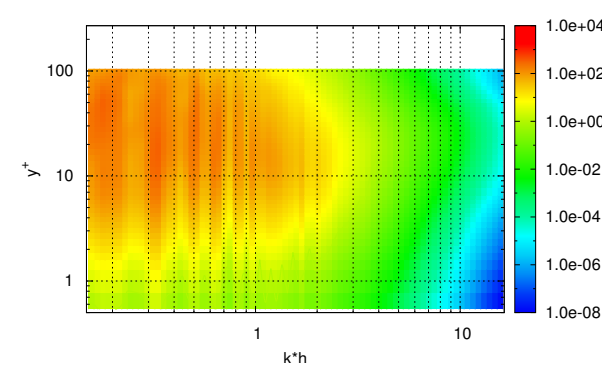

(e) Hot side $R e_{\tau}^{m}=180$

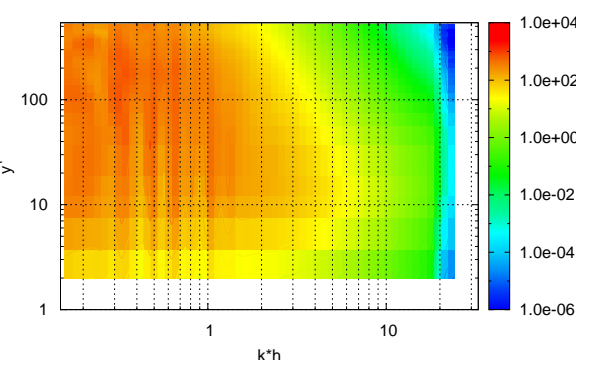

(b) Cold side $R e_{\tau}^{m}=395$

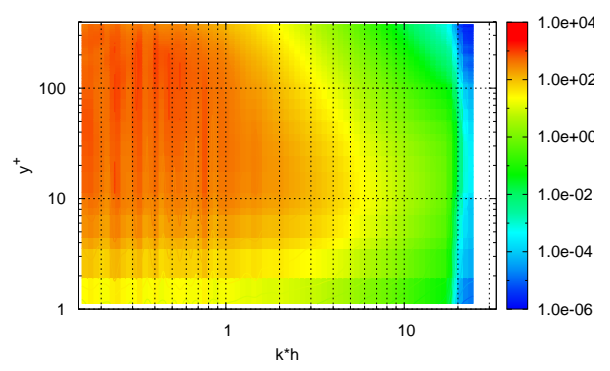

(d) Isothermal $R e_{\tau}=395$

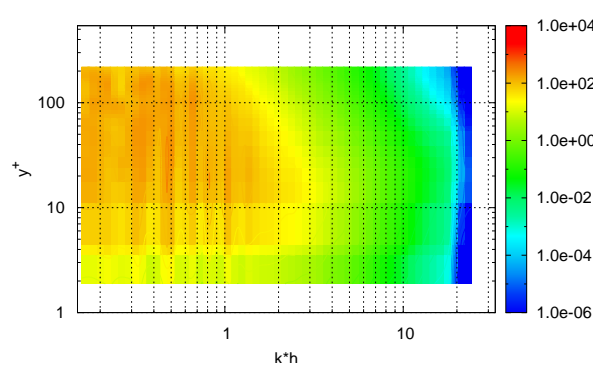

(f) Hot side $R e_{\tau}^{m}=395$

Figure 7: Turbulent kinetic energy, divided by $U_{\tau} \nu$. 


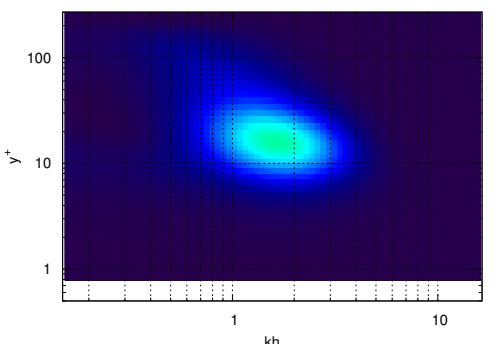

(a) Cold side $R e_{\tau}^{m}=180$

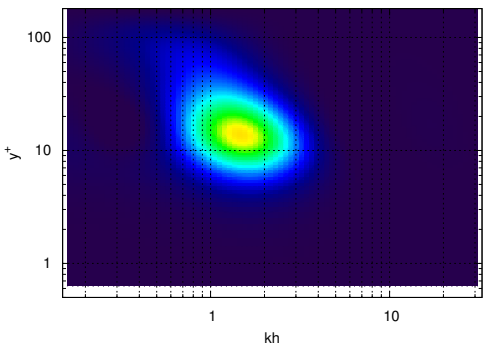

(c) Isothermal $R e_{\tau}=180$

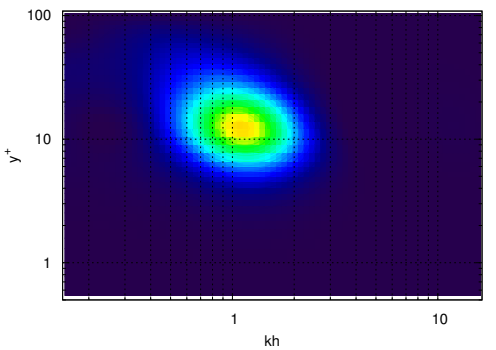

(e) Hot side $R e_{\tau}^{m}=180$

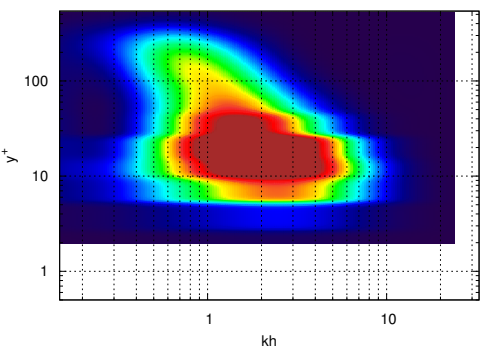

(b) Cold side $R e_{\tau}^{m}=395$

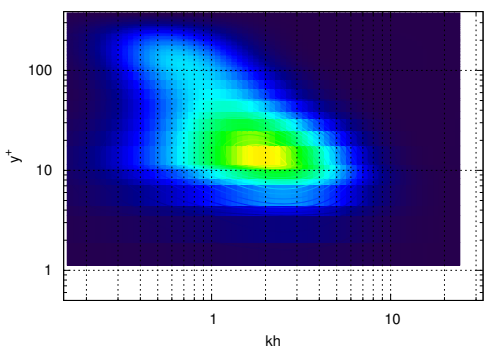

(d) Isothermal $R e_{\tau}=395$

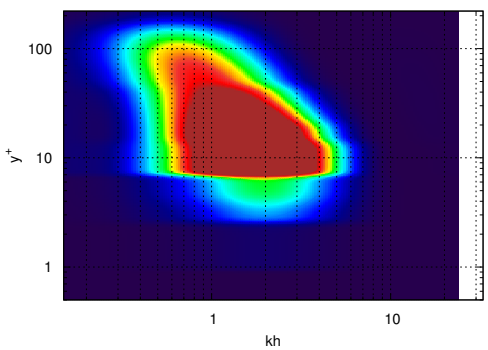

(f) Hot side $R e_{\tau}^{m}=395$

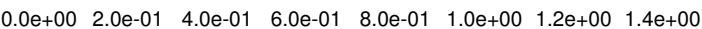

Figure 8: Turbulent production term. 


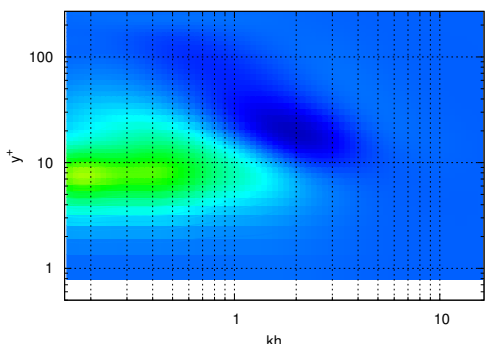

(a) Cold side $R e_{\tau}^{m}=180$

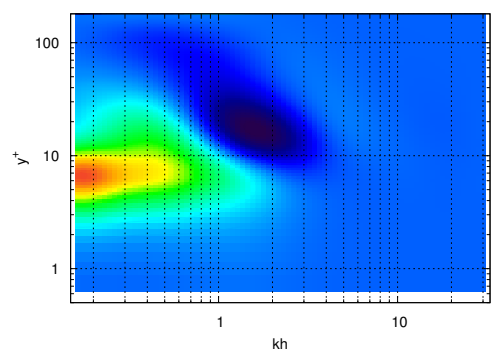

(c) Isothermal $R e_{\tau}=180$

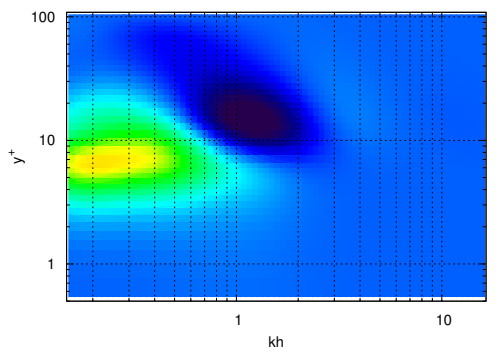

(e) Hot side $R e_{\tau}^{m}=180$

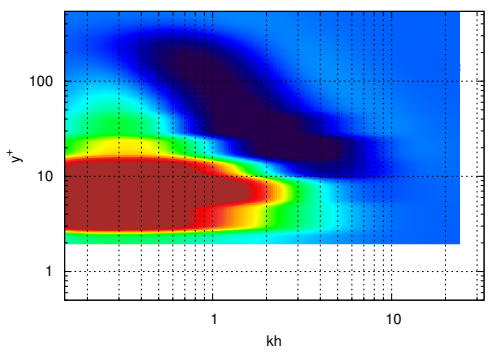

(b) Cold side $R e_{\tau}^{m}=395$

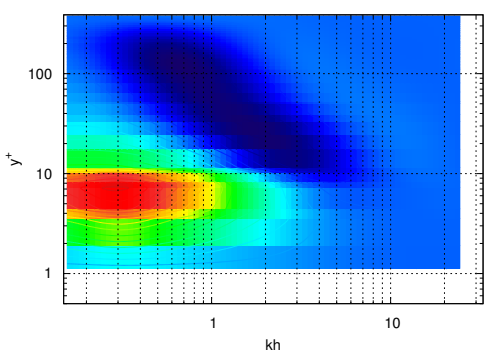

(d) Isothermal $R e_{\tau}=395$

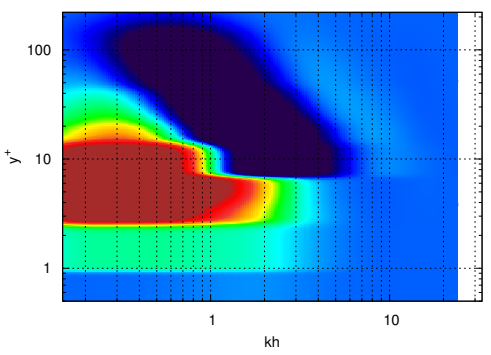

(f) Hot side $R e_{\tau}^{m}=395$

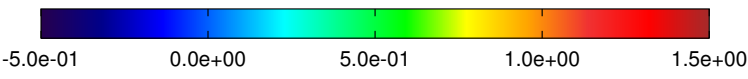

Figure 9: Total transfer (total triadic term and total pressure term). 


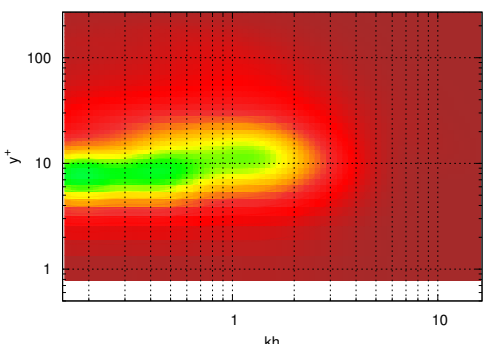

(a) Cold side $R e_{\tau}^{m}=180$

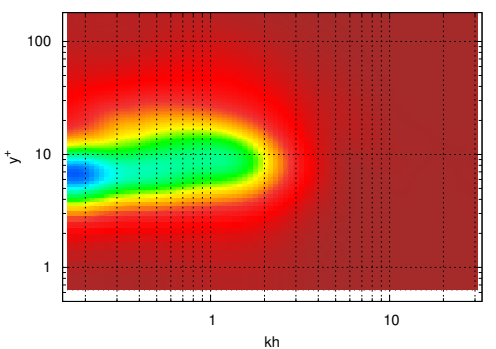

(c) Isothermal $R e_{\tau}=180$

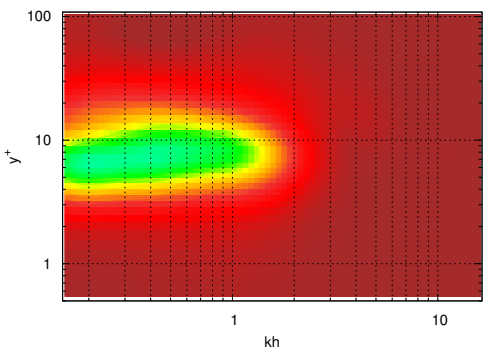

(e) Hot side $R e_{\tau}^{m}=180$

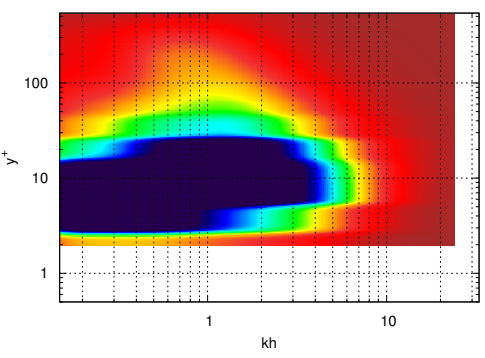

(b) Cold side $R e_{\tau}^{m}=395$

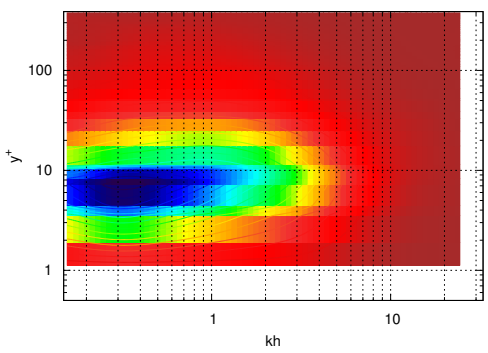

(d) Isothermal $R e_{\tau}=395$

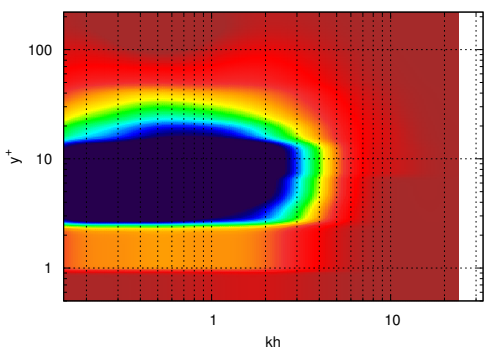

(f) Hot side $R e_{\tau}^{m}=395$

$-1.4 \mathrm{e}+00-1.2 \mathrm{e}+00-1.0 \mathrm{e}+00-8.0 \mathrm{e}-01-6.0 \mathrm{e}-01 \quad-4.0 \mathrm{e}-01 \quad-2.0 \mathrm{e}-01 \quad 0.0 \mathrm{e}+00$

Figure 10: Viscous effect term. 


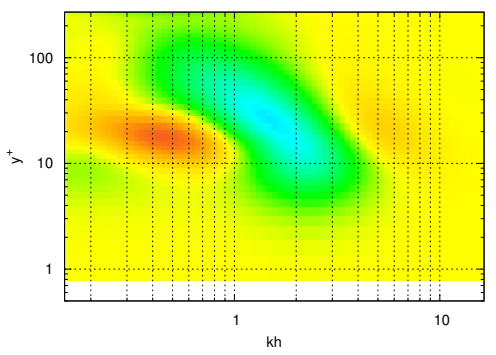

(a) Cold side $R e_{\tau}^{m}=180$

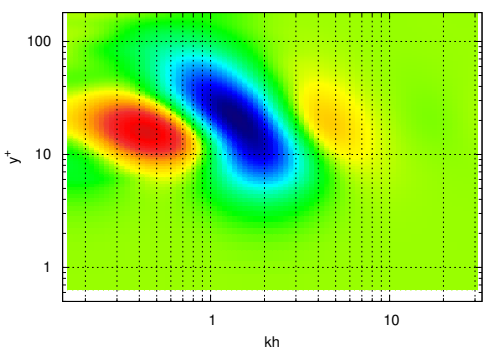

(c) Isothermal $R e_{\tau}=180$

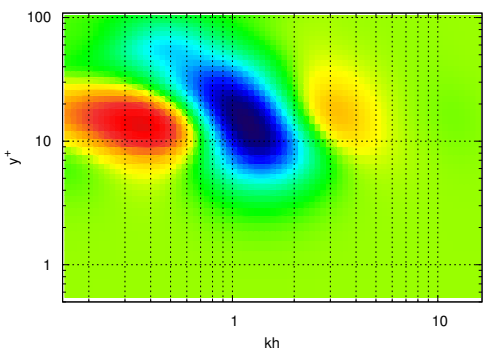

(e) Hot side $R e_{\tau}^{m}=180$

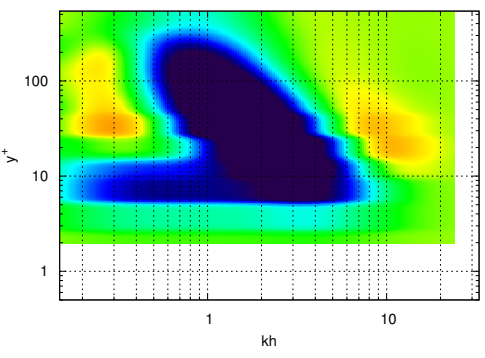

(b) Cold side $R e_{\tau}^{m}=395$

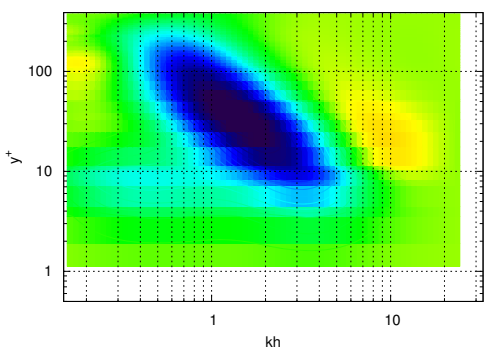

(d) Isothermal $R e_{\tau}=395$

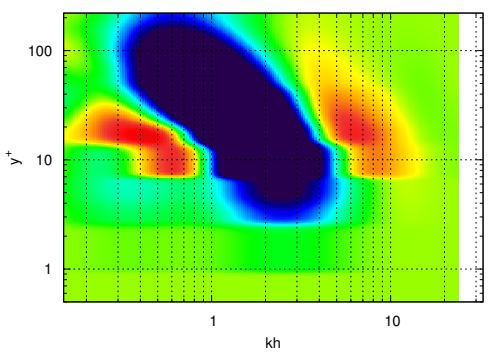

(f) Hot side $R e_{\tau}^{m}=395$

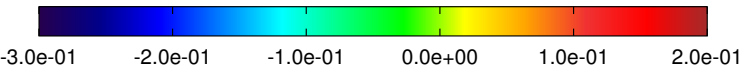

Figure 11: Triadic in-plane term. 


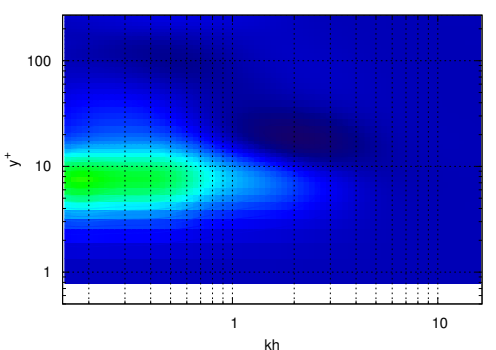

(a) Cold side $R_{\tau}^{m}=180$

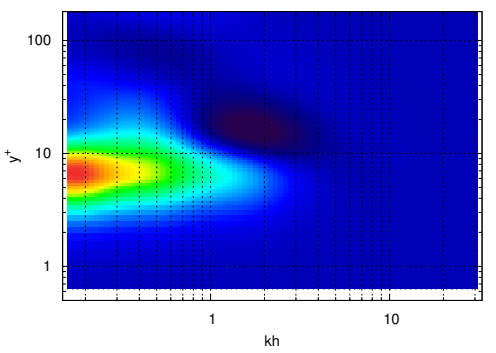

(c) Isothermal $R e_{\tau}=180$

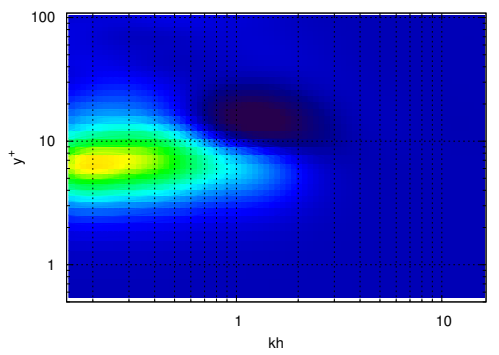

(e) Hot side $R e_{\tau}^{m}=180$

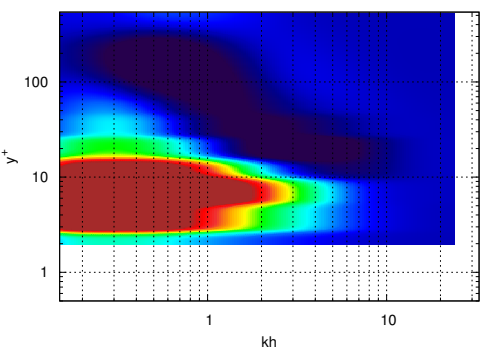

(b) Cold side $R e_{\tau}^{m}=395$

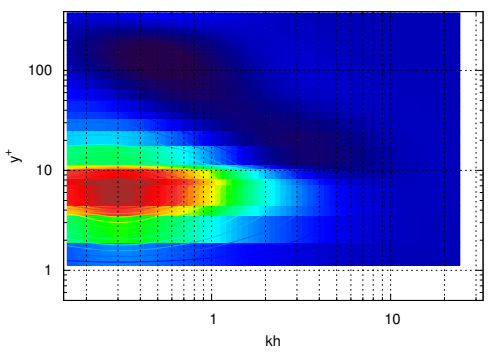

(d) Isothermal $R e_{\tau}=395$

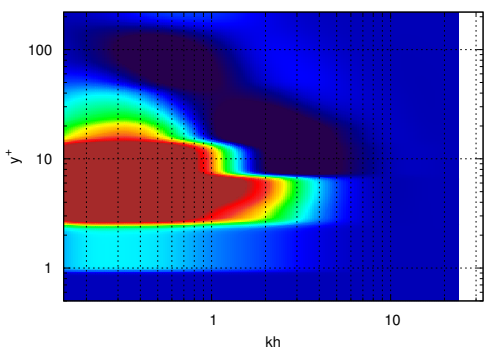

(f) Hot side $R e_{\tau}^{m}=395$

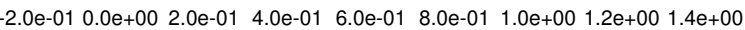

Figure 12: Triadic inter-plane term. 


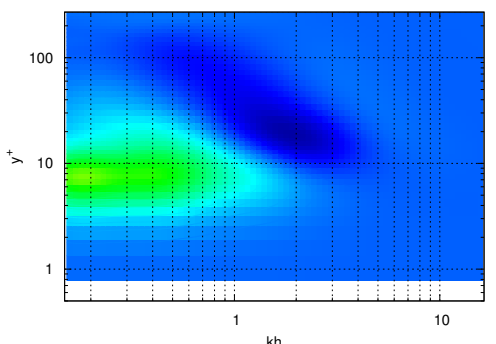

(a) Cold side $R e_{\tau}^{m}=180$

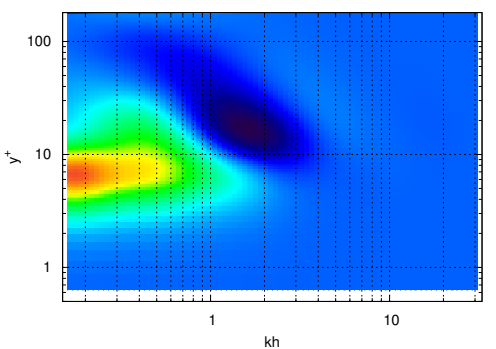

(c) Isothermal $R e_{\tau}=180$

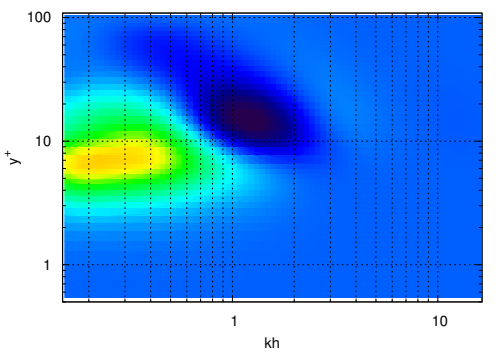

(e) Hot side $R e_{\tau}^{m}=180$

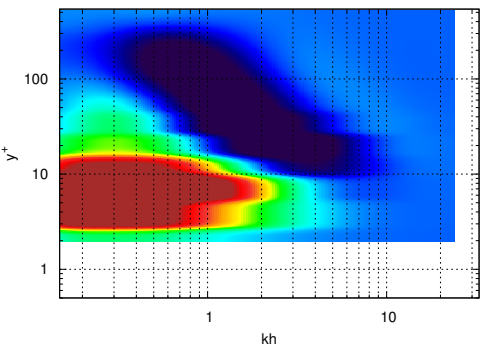

(b) Cold side $R e_{\tau}^{m}=395$

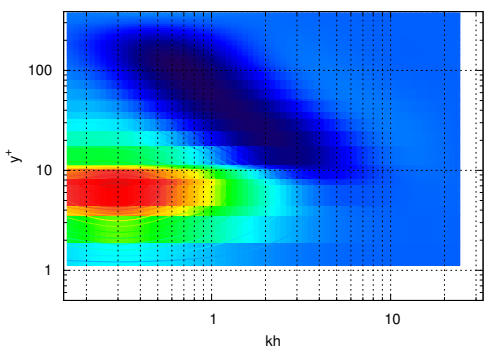

(d) Isothermal $R e_{\tau}=395$

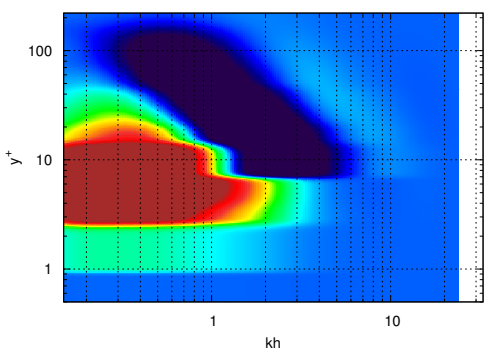

(f) Hot side $R e_{\tau}^{m}=395$

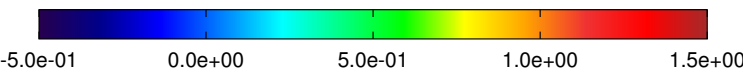

Figure 13: Total triadic term. 


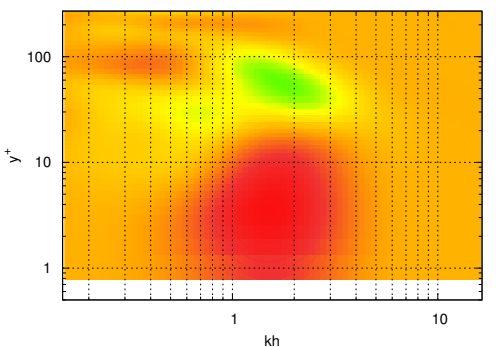

(a) Cold side $R e_{\tau}^{m}=180$

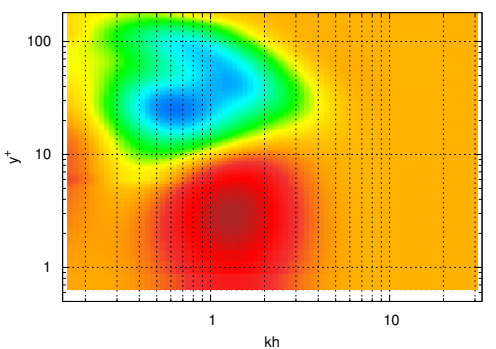

(c) Isothermal $R e_{\tau}=180$

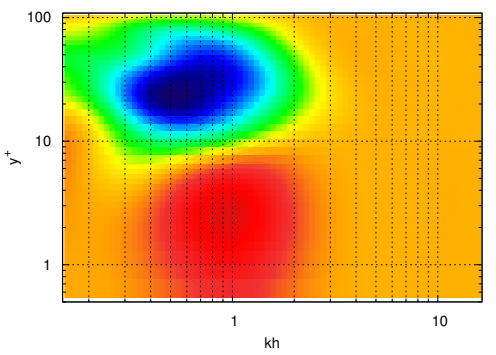

(e) Hot side $R e_{\tau}^{m}=180$

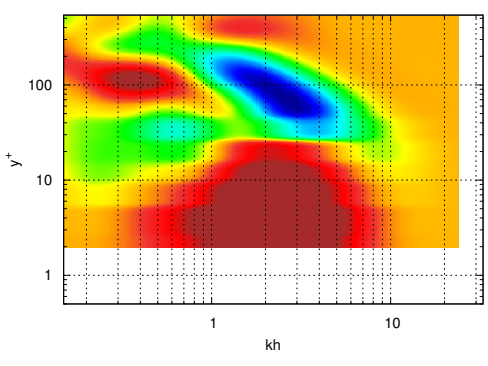

(b) Cold side $R e_{\tau}^{m}=395$

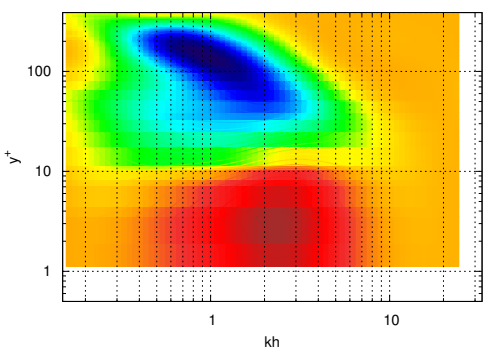

(d) Isothermal $R e_{\tau}=395$

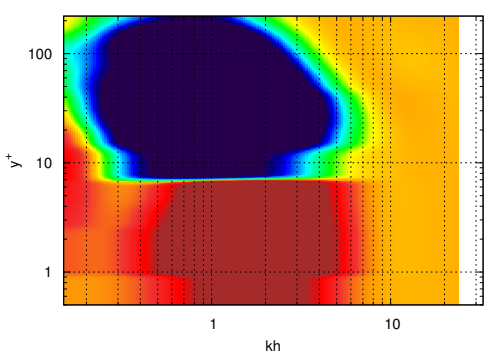

(f) Hot side $R e_{\tau}^{m}=395$

Figure 14: Pressure in-plane term. 


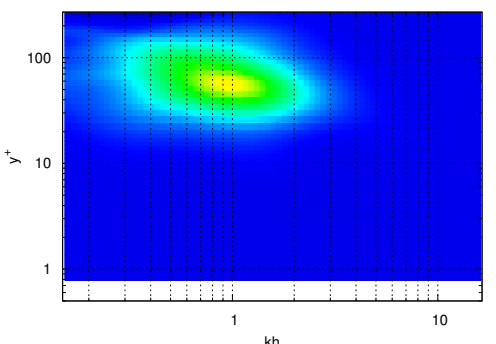

(a) Cold side $R e_{\tau}^{m}=180$

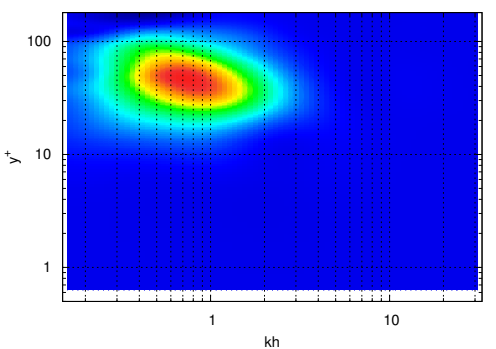

(c) Isothermal $R e_{\tau}=180$

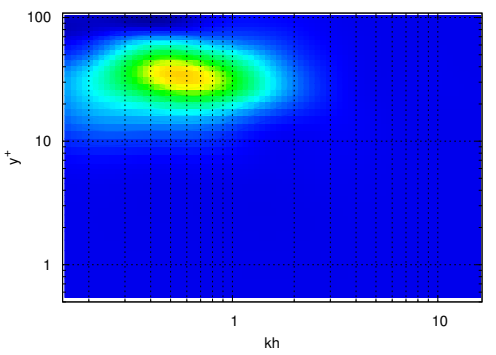

(e) Hot side $R e_{\tau}^{m}=180$

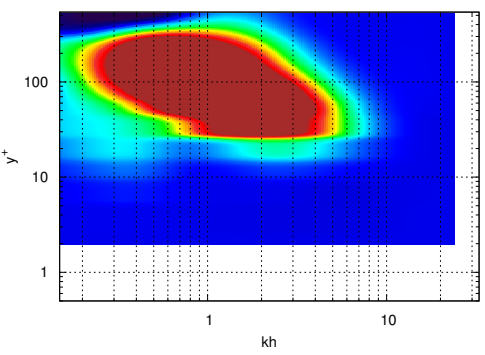

(b) Cold side $R e_{\tau}^{m}=395$

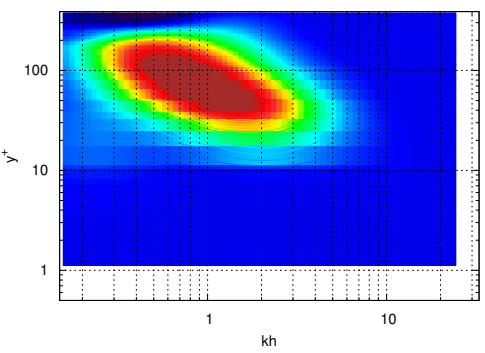

(d) Isothermal $R e_{\tau}=395$

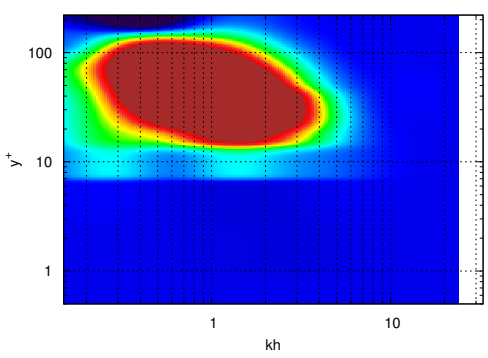

(f) Hot side $R e_{\tau}^{m}=395$

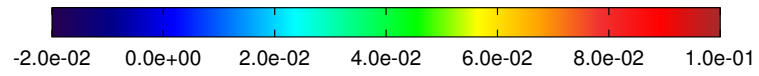

Figure 15: Pressure inter-plane term. 


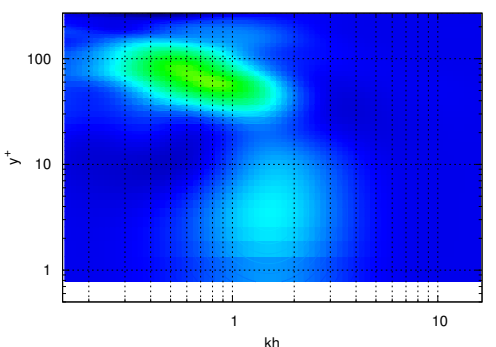

(a) Cold side $R e_{\tau}^{m}=180$

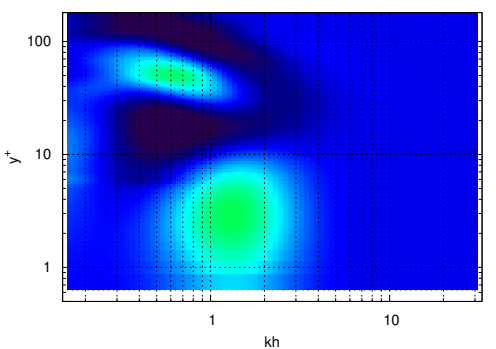

(c) Isothermal $R e_{\tau}=180$

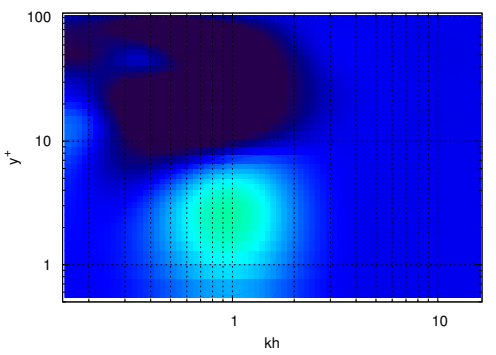

(e) Hot side $R e_{\tau}^{m}=180$

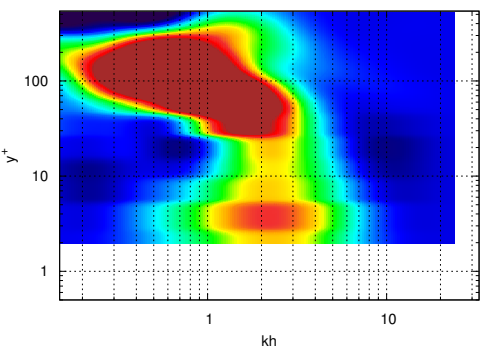

(b) Cold side $R e_{\tau}^{m}=395$

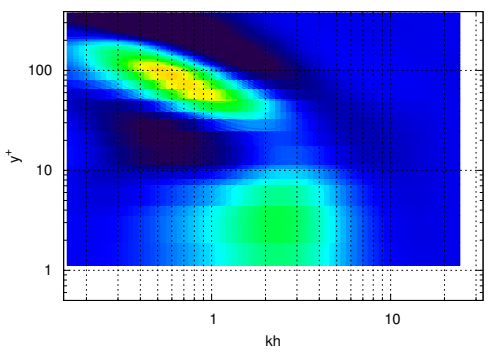

(d) Isothermal $R e_{\tau}=395$

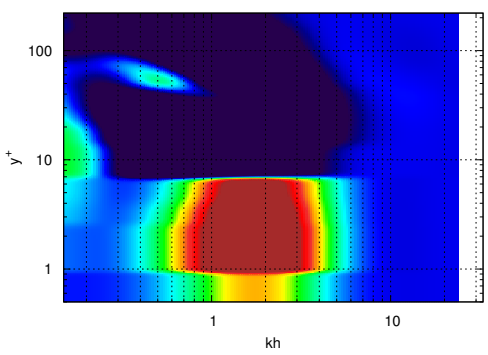

(f) Hot side $R e_{\tau}^{m}=395$

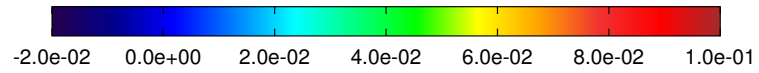

Figure 16: Total pressure term. 


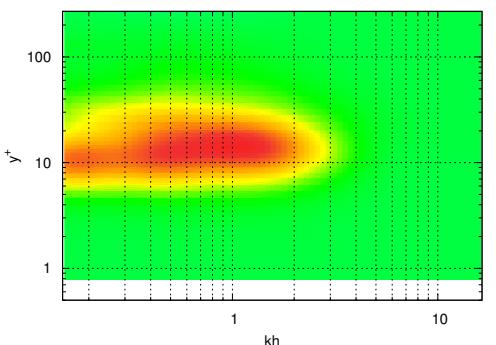

(a) Cold side $R e_{\tau}^{m}=180$

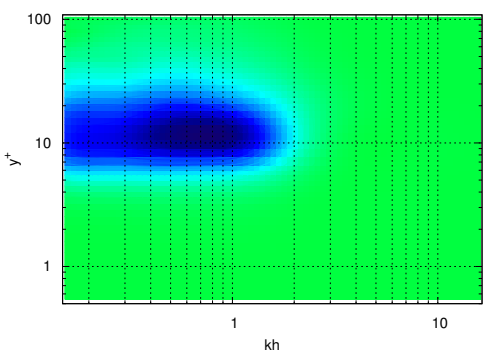

(c) Hot side $R e_{\tau}^{m}=180$

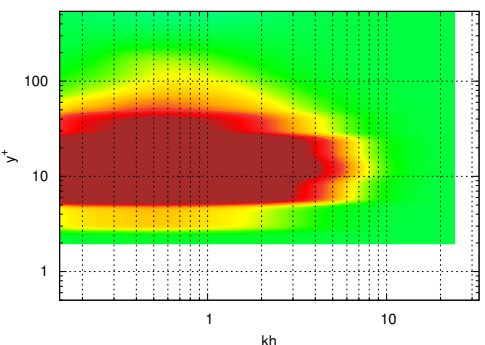

(b) Cold side $R e_{\tau}^{m}=395$

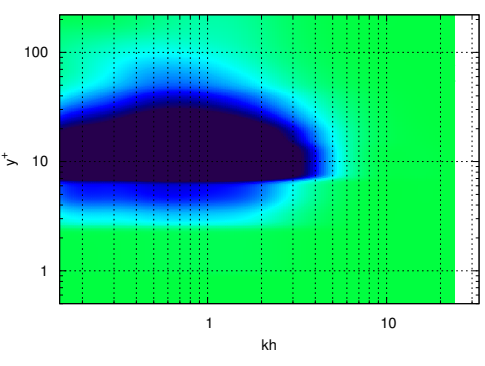

(d) Hot side $R e_{\tau}^{m}=395$

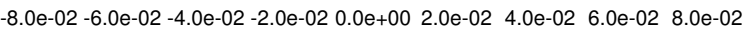

Figure 17: Thermal effects term. 
Appendix A. Derivation of the spectral turbulent kinetic energy evo-

\section{lution equation}

We apply a Fourier transform on equation (17). Taking into account the nonperiodicity of $y$ direction, the Fourier operator (A.1) is a 2D Fourier transform in the $x$ and $z$ directions. The Fourier transform of a function $g(x, y, z)$ is denoted $F_{k}^{y}\{g(x, y, z)\}$ or $\widehat{g}(\underline{k}, y)$ and defined as:

$$
F_{k}^{y}\{g(x, y, z)\}=\widehat{g}(\underline{k}, y)=\frac{1}{L_{x} L_{z}} \int_{0}^{L_{x}} \int_{0}^{L_{z}} g(x, y, z) \exp (-i \underline{k} \cdot \underline{x}) d x d z
$$

where $\underline{k}=\left(k_{x}, k_{z}\right)$ is the $2 \mathrm{D}$ wave vector and $\underline{x}=(x, z)$ is the $2 \mathrm{D}$ position vector. Let us apply this operator on equation (17):

$\frac{\partial \widehat{V}_{i}}{\partial t}=F_{k}^{y}\left\{-\frac{\partial V_{i} U_{j}}{\partial x_{j}}+\frac{1}{2} V_{i} \frac{\partial U_{j}}{\partial x_{j}}-b \frac{\partial P}{\partial x_{i}}\right\}+F_{k}^{y}\left\{b \frac{\partial}{\partial x_{j}}\left[\mu\left(\frac{\partial U_{i}}{\partial x_{j}}+\frac{\partial U_{j}}{\partial x_{i}}\right)\right]-b \frac{2}{3} \frac{\partial}{\partial x_{i}}\left(\mu \frac{\partial U_{j}}{\partial x_{j}}\right)\right\}$

Using a classical Reynolds decomposition, we split the velocity between mean part and fluctuation part $\left(V_{i}=\left\langle V_{i}\right\rangle+v_{i}^{\prime}\right)$. In addition, the flow homogeneity gives $\left(\left\langle U_{z}\right\rangle=0, \frac{\partial\langle f\rangle}{\partial x}=\frac{\partial\langle f\rangle}{\partial z}=0\right)$. Noticing that:

$$
\begin{aligned}
\frac{\partial \widehat{V_{i}}}{\partial t} & =\frac{\partial \widehat{v_{i}^{\prime}}}{\partial t} \\
F_{k}^{y}\left\{\frac{\partial V_{i} U_{j}}{\partial x_{j}}\right\} & =\left\langle V_{i}\right\rangle \frac{\widehat{\partial u_{j}^{\prime}}}{\partial x_{j}}+\widehat{v_{i}^{\prime}} \frac{\partial\left\langle U_{y}\right\rangle}{\partial y}+\widehat{u_{y}^{\prime}} \frac{\partial\left\langle V_{i}\right\rangle}{\partial y}+\left\langle U_{j}\right\rangle \frac{\widehat{\partial v_{i}^{\prime}}}{\partial x_{j}}+F_{k}^{y}\left\{\frac{\partial v_{i}^{\prime} u_{j}^{\prime}}{\partial x_{j}}\right\} \\
F_{k}^{y}\left\{V_{i} \frac{\partial U_{j}}{\partial x_{j}}\right\} & =\left\langle V_{i}\right\rangle \frac{\widehat{\partial u_{j}^{\prime}}}{\partial x_{j}}+\widehat{v_{i}^{\prime}} \frac{\partial\left\langle U_{y}\right\rangle}{\partial y}+\widehat{v_{i}^{\prime}} \frac{\partial u_{j}^{\prime}}{\partial x_{j}}
\end{aligned}
$$

we obtain:

$$
\begin{array}{r}
\frac{\partial \widehat{v_{i}^{\prime}}}{\partial t}=-\widehat{u_{y}^{\prime}} \frac{\partial\left\langle V_{i}\right\rangle}{\partial y}-\left\langle U_{j}\right\rangle \frac{\widehat{\partial v_{i}^{\prime}}}{\partial x_{j}}-\frac{1}{2}\left(\left\langle V_{i}\right\rangle \frac{\widehat{\partial u_{j}^{\prime}}}{\partial x_{j}}+\widehat{v_{i}^{\prime}} \frac{\partial\left\langle U_{y}\right\rangle}{\partial y}-\widehat{v_{i}^{\prime}} \frac{\widehat{\partial u_{j}^{\prime}}}{\partial x_{j}}\right)-F_{k}^{y}\left\{\frac{\partial v_{i}^{\prime} u_{j}^{\prime}}{\partial x_{j}}\right\}-b \frac{\widehat{\partial P}}{\partial x_{i}} \\
+F_{k}^{y}\left\{b \frac{\partial}{\partial x_{j}}\left[\mu\left(\frac{\partial U_{i}}{\partial x_{j}}+\frac{\partial U_{j}}{\partial x_{i}}\right)\right]-b \frac{2}{3} \frac{\partial}{\partial x_{i}}\left(\mu \frac{\partial U_{j}}{\partial x_{j}}\right)\right\}
\end{array}
$$


Following, we multiply equation (A.3) by ${\widehat{v_{i}^{\prime}}}^{*}$, where $\widehat{g}^{*}$ is the complex conjugate of $\widehat{g}$, and sum the equation on $i$ subscript.

$$
\begin{array}{r}
{\widehat{v_{i}^{\prime}}}^{*} \frac{\partial{\widehat{v_{i}^{\prime}}}_{\partial t}}{\partial t}-{\widehat{v_{i}^{\prime}}}^{*} \widehat{u_{y}^{\prime}} \frac{\partial\left\langle V_{i}\right\rangle}{\partial y}-{\widehat{v_{i}^{\prime}}}^{*}\left\langle U_{j}\right\rangle \frac{\widehat{\partial v_{i}^{\prime}}}{\partial x_{j}}-\frac{{\widehat{v_{i}^{\prime}}}^{*}}{2}\left(\left\langle V_{i}\right\rangle \frac{\widehat{\partial u_{j}^{\prime}}}{\partial x_{j}}+\widehat{v_{i}^{\prime}} \frac{\partial\left\langle U_{y}\right\rangle}{\partial y}-\widehat{v_{i}^{\prime}} \frac{\widehat{\partial u_{j}^{\prime}}}{\partial x_{j}}\right) \\
-{\widehat{v_{i}^{\prime}}}^{*} F_{k}^{y}\left\{\frac{\partial v_{i}^{\prime} u_{j}^{\prime}}{\partial x_{j}}\right\}-{\widehat{v_{i}^{\prime}}}^{*} b \frac{\partial P}{\partial x_{i}}+{\widehat{v_{i}^{\prime}}}^{*} F_{k}^{y}\left\{b \frac{\partial}{\partial x_{j}}\left[\mu\left(\frac{\partial U_{i}}{\partial x_{j}}+{\widehat{v_{i}^{\prime}}}^{*} \frac{\partial U_{j}}{\partial x_{i}}\right)\right]-b \frac{2}{3} \frac{\partial}{\partial x_{i}}\left(\mu \frac{\partial U_{j}}{\partial x_{j}}\right)\right\}
\end{array}
$$

Noticing that:

$$
\sum_{i} \Re\left[\widehat{v_{i}^{\prime}} * \frac{\partial \widehat{v_{i}^{\prime}}}{\partial t}\right]=\sum_{i} \frac{1}{2} \frac{\partial \widehat{v_{i}^{\prime}} * \widehat{v_{i}^{\prime}}}{\partial t}=\frac{\partial E_{c}}{\partial t}
$$

where $E_{c}=\frac{1}{2}{\widehat{v_{i}^{\prime}}}_{i}^{*}{\widehat{v_{i}^{\prime}}}_{i}$ is the instantaneous turbulent kinetic energy (TKE) in the spectral space and $\Re$ is the real part of a complex number, we obtain the spectral equation of the instantaneous turbulent kinetic energy evolution:

$$
\begin{aligned}
\frac{\partial E_{c}}{\partial t}= & \Re\left[-{\widehat{v_{i}^{\prime}}}^{*} \widehat{u_{y}^{\prime}} \frac{\partial\left\langle V_{i}\right\rangle}{\partial y}-{\widehat{v_{i}^{\prime}}}^{*} F_{k}^{y}\left\{\frac{\partial v_{i}^{\prime} u_{j}^{\prime}}{\partial x_{j}}\right\}-{\widehat{v_{i}^{\prime}}}^{*} b \frac{\widehat{\partial P}}{\partial x_{i}}\right. \\
& \left.\left.\left.\left.-{\widehat{v_{i}^{\prime}}}^{*}\left(\left\langle V_{i}\right\rangle \frac{\widehat{\partial u_{j}^{\prime}}}{\partial x_{j}}+\widehat{v_{i}^{\prime}} \frac{\partial\left\langle U_{y}\right\rangle}{\partial y}-\widehat{v_{i}^{\prime}} \frac{\widehat{\partial u_{j}^{\prime}}}{\partial x_{j}}\right)-{\widehat{v_{i}^{\prime}}}^{*}\left\langle U_{y}\right\rangle \frac{\partial{\widehat{v_{i}^{\prime}}}_{\partial y}}{\partial x_{i}}\right)\right]-b \frac{2}{3} \frac{\partial}{\partial x_{i}}\left(\mu \frac{\partial U_{j}}{\partial x_{j}}\right)\right\}\right] \\
& +{\widehat{v_{i}^{\prime}}}^{*} F_{k}^{y}\left\{b \frac{\partial}{\partial x_{j}}\left[\mu\left(\frac{\partial U_{i}}{\partial x_{j}}+\frac{\partial U_{j}}{\partial x_{i}}\right)\right]\right.
\end{aligned}
$$

Note that $\Re\left[{\widehat{v_{i}^{\prime}}}^{*}\left\langle U_{x}\right\rangle \frac{\widehat{\partial v_{i}^{\prime}}}{\partial x}\right]$ is equal to zero, because ${\widehat{v_{i}^{\prime}}}^{*}\left\langle U_{x}\right\rangle \frac{\widehat{\partial v_{i}^{\prime}}}{\partial x}$ is a pure imaginary number. It remains only $\Re\left[\widehat{v}_{i}^{*}\left\langle U_{y}\right\rangle \frac{\partial \widehat{v_{i}^{\prime}}}{\partial y}\right]$.

The spectral equation (A.6) of the instantaneous TKE evolution depends on the four parameters $k_{x}, k_{z}, y$ and $t$. In order to simplify the interpretation of the terms, we integrate the 2D Fourier space following the wave vector $\underline{k}$ norms:

$$
f(k, y)=\int_{k=\|\underline{k}\|} f\left(k_{x}, k_{z}, y\right) d \underline{k}
$$

\section{0}

[1] X. Daguenet-Frick, J.-M. Foucaut, S. Coudert, A. Toutant, G. Olalde, Experimental analysis of the turbulent flow behavior of a textured surface 
proposed for asymmetric heat exchangers, Flow, turbulence and combustion 89 (1) (2012) 149-169.

[2] A. Toutant, F. Bataille, Turbulence statistics in a fully developed channel flow submitted to a high temperature gradient, International Journal of Thermal Sciences 74 (0) (2013) 104-118.

[3] P. Chassaing, R. Antonia, F. Anselmet, L. Joly, S. Sarkar, Variable density fluid turbulence, Vol. 69, Springer Science \& Business Media, 2013.

[4] J. A. Domaradzki, R. S. Rogallo, Local energy transfer and nonlocal interactions in homogeneous, isotropic turbulence, Physics of Fluids A: Fluid Dynamics (1989-1993) 2 (3) (1990) 413-426.

[5] Y. Zhou, Degrees of locality of energy transfer in the inertial range, Physics of Fluids A: Fluid Dynamics (1989-1993) 5 (5) (1993) 1092-1094.

[6] Y. Zhou, Interacting scales and energy transfer in isotropic turbulence, Physics of Fluids A: Fluid Dynamics (1989-1993) 5 (10) (1993) 2511-2524.

[7] J. A. Domaradzki, W. Liu, C. Hartel, L. Kleiser, Energy transfer in numerically simulated wall-bounded turbulent flows, Physics of Fluids 6 (4) (1994) 1583-1599.

[8] N. Marati, C. Casciola, R. Piva, Energy cascade and spatial fluxes in wall turbulence, Journal of Fluid Mechanics 521 (2004) 191-215.

[9] I. A. Bolotnov, R. T. L. Jr., D. A. Drew, K. E. Jansen, A. A. Oberai, Spectral analysis of turbulence based on the $\{\mathrm{DNS}\}$ of a channel flow, Computers and Fluids 39 (4) (2010) 640 - 655.

[10] A. Cimarelli, E. De Angelis, The physics of energy transfer toward improved subgrid-scale models, Physics of Fluids (1994-present) 26 (5) (2014) 055103.

[11] A. Cimarelli, E. De Angelis, C. Casciola, Paths of energy in turbulent channel flows, Journal of Fluid Mechanics 715 (2013) 436-451. 
[12] A. Favre, Statistical equations of turbulent gases, Problems of hydrodynamics and continuum mechanics (1969) 231-266.

[13] D. C. Wilcox, et al., Turbulence modeling for CFD, Vol. 2, DCW industries La Canada, CA, 1998.

[14] P. Huang, G. Coleman, P. Bradshaw, Compressible turbulent channel flows: Dns results and modelling, Journal of Fluid Mechanics 305 (1995) 185-218.

[15] P. Chassaing, Une alternative à la formulation des équations du mouvement turbulent d'un fluide à masse volumique variable, Journal de mécanique théorique et appliquée 4 (3) (1985) 375-389.

[16] F. Aulery, A. Toutant, F. Bataille, Y. Zhou, Energy transfer process of anisothermal wall-bounded flows, Physics Letters A 379 (24) (2015) 15201526.

[17] C.-S. Yih, Exact solutions for steady two-dimensional flow of a stratified fluid, Journal of Fluid Mechanics 9 (02) (1960) 161-174.

[18] S. Kida, S. Orszag, Energy and spectral dynamics in decaying compressible turbulence, Journal of Scientific Computing 7 (1) (1992) 1-34.

[19] J. Rodriguez Azara, G. Emanuel, Compressible rotational flows generated by the substitution principle, Physics of Fluids 31 (1988) 1058-1063.

[20] A. W. Cook, Y. Zhou, Energy transfer in rayleigh-taylor instability, Phys. Rev. E 66 (2002) 026312.

[21] A. Kolmogorov, Cr acad. sci., ussr 30301 kolmogorov an 1941, CR Acad. Sci., USSR 30 (1941) 538.

[22] S. Serra, A. Toutant, F. Bataille, Y. Zhou, Turbulent kinetic energy spectrum in very anisothermal flows, Physics Letters A 376 (45) (2012) 31773184 . 
[23] Y. Zhou, W. Matthaeus, P. Dmitruk, Colloquium: Magnetohydrodynamic turbulence and time scales in astrophysical and space plasmas, Reviews of Modern Physics 76 (4) (2004) 1015.

[24] Y. Zhou, Renormalization group theory for fluid and plasma turbulence, Physics Reports 488 (1) (2010) 1-49.

[25] Y. Zhou, S. Oughton, Nonlocality and the critical reynolds numbers of the minimum state magnetohydrodynamic turbulence, Physics of Plasmas (1994-present) 18 (7) (2011) 072304.

[26] S. Paolucci, On the filtering of sound from the Navier-Stokes equations, Tech. Rep. SAND82-8257, Sandia National Labs., Livermore, CA (USA) (1982).

[27] R. Courant, K. Friedrichs, H. Lewy, Über die partiellen Differenzengleichungen der mathematischen Physik, Mathematische Annalen 100 (1) (1928) 32-74.

[28] E. Garnier, N. Adams, P. Sagaut, Large eddy simulation for compressible flows, Springer Science \& Business Media, 2009.

[29] F. Nicoud, F. Ducros, Subgrid-scale stress modelling based on the square of the velocity gradient tensor, Flow, Turbulence and Combustion 62 (3) (1999) 183-200.

[30] T. M. Eidson, Numerical simulation of the turbulent Rayleigh-Bénard problem using subgrid modelling, Journal of Fluid Mechanics 158 (1985) $245-268$.

[31] W. Sutherland, LII. the viscosity of gases and molecular force, The London, Edinburgh, and Dublin Philosophical Magazine and Journal of Science 36 (223) (1893) 507-531.

[32] B. Leonard, A stable and accurate convective modelling procedure based on quadratic upstream interpolation, Computer Methods in Applied Mechanics and Engineering 19 (1) (1979) 59-98. 
[33] C. Calvin, O. Cueto, P. Emonot, An object-oriented approach to the design of fluid mechanics software, ESAIM: Mathematical Modelling and Numerical Analysis 36 (05) (2002) 907-921.

[34] M. Frigo, S. G. Johnson, FFTW: An adaptive software architecture for the FFT, in: Acoustics, Speech and Signal Processing, 1998. Proceedings of the 1998 IEEE International Conference on, Vol. 3, IEEE, 1998, pp. 1381-1384.

[35] J. Kim, P. Moin, R. Moser, Turbulence statistics in fully developed channel flow at low reynolds number, Journal of Fluid Mechanics 177 (1987) 133166.

[36] R. D. Moser, J. Kim, N. N. Mansour, Direct numerical simulation of turbulent channel flow up to $r e_{\tau}=590$, Physics of Fluids 11 (4) (1999) 943-945.

[37] U. Piomelli, Y. Yu, R. J. Adrian, Subgrid-scale energy transfer and nearwall turbulence structure, Physics of Fluids 8 (1) (1996) 215-224.

[38] A.-T. Le, G. N. Coleman, J. Kim, Near-wall turbulence structures in threedimensional boundary layers, International Journal of Heat and Fluid Flow 21 (5) (2000) $480-488$.

[39] A. Cimarelli, E. D. Angelis, Anisotropic dynamics and sub-grid energy transfer in wall-turbulence, Physics of Fluids 24 (1) (2012) 015102.

530 [40] P. Chassaing, Turbulence en mécanique des fluides, Cépaduès-éditions, 2000. 